\title{
Application of a Computational Glass Model to the Shock Response of Soda-Lime Glass
}

\author{
Joshua E. Gorfain ${ }^{1} \cdot$ Christopher T. Key ${ }^{2}$ C. Scott Alexander ${ }^{3}$
}

Received: 5 January 2016/Accepted: 11 April 2016/Published online: 20 April 2016

(C) Society for Experimental Mechanics, Inc 2016

\begin{abstract}
This article details the implementation and application of the glass-specific computational constitutive model by Holmquist and Johnson (J Appl Mech 78:051003, 2011) to simulate the dynamic response of soda-lime glass under high rate and high pressure shock conditions. The predictive capabilities of this model are assessed through comparison of experimental data with numerical results from computations using the CTH shock physics code. The formulation of this glass model is reviewed in the context of its implementation within $\mathrm{CTH}$. Using a variety of experimental data compiled from the open literature, a complete parameterization of the model describing the observed behavior of soda-lime glass is developed. Simulation results using the calibrated soda-lime glass model are compared to flyer plate and Taylor rod impact experimental data covering a range of impact and failure conditions spanning an order of magnitude in velocity and pressure. The complex behavior observed in the experimental testing is captured well in the computations, demonstrating the capability of the glass model within CTH.
\end{abstract}

Keywords Soda-lime glass - Shock physics - Impact · Failure $\cdot$ Constitutive modeling

Joshua E. Gorfain

jgorfain@aphysci.com

1 Applied Physical Sciences Corp., 4301 North Fairfax Drive, Suite 640, Arlington, VA 22203, USA

2 Applied Physical Sciences Corp., 475 Bridge Street, Suite 100, Groton, CT 06340, USA

3 Sandia National Laboratories, P.O. Box 5800, Albuquerque, NM 87185, USA

\section{Introduction}

Soda-lime glass is a commercially available plate (float) glass material utilized in a wide variety of commercial and military applications. Used primarily as a transparent window material, it is often used as a lower-cost surrogate for more expensive types of glass. Due to its widespread application, soda-lime glass may be subjected to a variety of dynamic conditions for which its performance is of interest.

Currently, there is a large research interest in glass, with the majority of efforts focused on borosilicate glass [2-12]. As a result of this extensive body of research, a large array of experimental data exists and computational models have been developed for borosilicate glass. A particular example is the new glass-specific constitutive model developed by Holmquist and Johnson [1]. While borosilicate and sodalime glass share many similarities in constitutive behavior, they have a number of significant differences as well. Most notably, soda-lime glass has a higher density and shock impedance but a lower dynamic strength [13-15]. Sodalime glass also exhibits a generally lower compressibility and permanent densification due to its partially filled structure. A complete loss of strength at the Hugoniot elastic limit (HEL) has also been observed, in contrast to borosilicate. Also, while borosilicate behaves similar to fused silica (pure $\mathrm{SiO}_{2}$ glass) in many ways, soda-lime exhibits more pronounced differences likely due to its lower $\mathrm{SiO}_{2}$ content and resultant changes to the glass structure [15].

The complex behavior of soda-lime glass and its widespread application favors the use of physics-based modeling to better understand experimentally observed phenomena. Design and application efforts similarly benefit from modeling as a more cost effective means of 
attaining novel and optimized products versus purely experimental trial-and-error approaches. Critical to realization of these benefits, a computational model must have a demonstrated accuracy providing confidence in the predicted results. Thus, the primary objective of this work is to assess the ability of the Holmquist and Johnson model, with properly calibrated parameters, to describe the dynamic response of soda-lime glass under high rate and high pressure conditions.

In this work, computational results will be compared to experimental data in order to evaluate the predictive capability of the model. First, the implementation of the glass model into the CTH shock physics code is described. Next, parameterization of the model characterizing the behavior of soda-lime glass is developed from a variety of experimental data compiled from the open literature. Finally, simulation results using the soda-lime glass model are compared to measured data from experiments covering a range of impact and failure conditions.

\section{Glass Model Description}

The glass model developed by Holmquist and Johnson of Southwest Research Institute [1], hereafter referred to as the SWRIG model, was recently implemented in the CTH shock physics code [16] developed by Sandia National Laboratories. CTH is a hydrocode designed for modeling complex multi-dimensional, multi-material problems characterized by large deformations and/or strong shocks. CTH utilizes an Eulerian framework to solve the finite volume approximations of the mass, momentum, and energy conservation equations.

Although presented in detail by Holmquist and Johnson, a brief description of the SWRIG model is repeated here with particular attention paid to the specifics of its implementation into CTH. It is important to note that all of the computations using the SWRIG model (by Holmquist and Johnson and other researchers) in the open literature to date were performed using EPIC; a Lagrangian finite element/particle code [17]. This work is the first reported use of this model in an Eulerian framework such as that of CTH.

\section{SWRIG Model in CTH}

The SWRIG model consists of three primary components: strength, damage, and an equation of state (EOS). The CTH implementation adds a fourth component, tensile fracture, which communicates with the other three components and is necessary to introduce voids properly within the Eulerian framework. The strength and damage components are used to compute the deviatoric stress tensor for the glass, while the EOS describes the hydrodynamic behavior and provides the necessary thermodynamic relationships. Plastic deformation in the model is treated using traditional J2 flow theory, while damage is characterized through an internal state variable, $D$, where a value of zero corresponds to an intact (virgin) material and a value of unity indicates material that is completely failed. In the model, the failed glass state is assumed as an effective continuum of fractured and comminuted material. Failure waves, previously observed in shock loaded glass, are thus modeled by propagating regions of failed glass. Damage provides a coupling between the strength and EOS associated with dilatancy (bulking) upon failure. Finally, strength (flow stress or the ability to support shear stress) is dependent on the condition of the material (intact or failed), its location (interior, surface, or adjacent to failed material), and the temperature, pressure, and strain rate.

\section{Strength Model}

The strength component of the SWRIG model provides a separate description of the intact strength (flow stress) of the glass depending on its location within the physical geometry. Graphical depiction of the strength is shown in Fig. 1. The reference strength corresponds to that of intact material adjacent to failed material (or material with a very rough finish) having a high defect density. Thus the reference strength is generally considered the weakest of the intact material locations and serves as the basis for which the strength of the remaining locations is determined. The intact strength of interior and surface materials are described separately such that increased interior strength and the effect of surface finish may be considered.

The CTH implementation automatically determines the appropriate location-dependent strength description from the model geometry and its evolution during the course of a computation. The location assignment for cells containing intact material are specified on input at the start of the computation. Unless specified differently by the user, all cells containing glass material is assumed to be initially intact. The exterior surface of the glass body is assigned the surface location as determined from the glass material volume fractions within the mesh, resulting in a surface material layer approximately one cell thick. The initially intact assumption precludes the presence of any reference (adjacent to failed) material, thus all glass not on the surface is interior, and is assigned this strength accordingly. At the end of each time-step, a searching algorithm looks at cells adjacent (in all directions) to those containing glass material to see if any have failed. If adjacent failed cells are found, then the location assigned to the current cell containing glass material is updated to adjacent to failed 

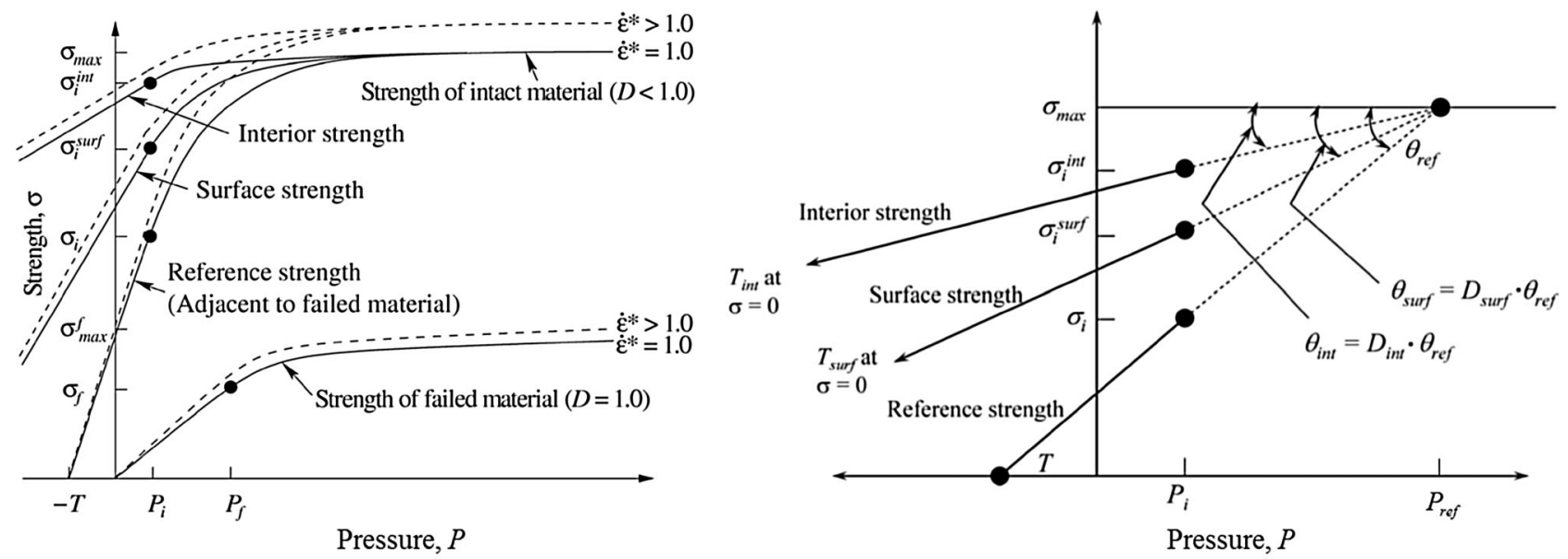

Fig. 1 Glass model strength description, reproduced with permission from ASME [1]

(reference). If no adjacent to failed cells are found, then the current intact location assignment is retained. These location assignments are tracked using integer-valued history variables which are advected with the material as it fluxes through the Eulerian grid. The algorithm includes logic designed to minimize and correct advection errors, taking advantage of the integer nature of these history variables before they are used by the SWRIG model to determine the material response.

The reference strength (for a strain rate of $\dot{\varepsilon}=1 \mathrm{~s}^{-1}$ ) varies linearly between zero at a tensile pressure of $-T$ and a strength of $\sigma_{i}$ at a pressure of $P_{i}$. At pressures greater than $P_{i}$, the strength is given by,

$\sigma=\sigma_{i}+\left(\sigma_{\max }-\sigma_{i}\right)\left\{1-\exp \left[-\alpha_{i}\left(P-P_{i}\right)\right]\right\}$,

where

$\alpha_{i}=\sigma_{i} /\left[\left(\sigma_{\max }-\sigma_{i}\right)\left(P_{i}+T\right)\right]$.

This strength form is a function of the current pressure, $P$, and asymptotically approaches a maximum compressive strength of $\sigma_{\max }$ at high pressures. The intact strength of surface and interior material is determined by factors $D_{\text {surf }}$ and $D_{\text {int }}$, which augment the reference strength [Eq. (1)] such that greater strength may be provided in the lower pressure/tensile regime $\left(P \leq \sim P_{i}\right)$ while the high pressure strength limit $\left(\sigma_{\max }\right)$ for all three material locations are identical. The relationship between the interior and surface strength curves and the $D_{\text {surf }}$ and $D_{\text {int }}$ factors is shown in Fig. 1, which is based upon the angle between the linear pressure-dependent $\left(P \leq P_{i}\right)$ and (high) pressure-independent maximum strength $\left(\sigma_{\max }\right)$ regions of the strength curves.

The strength of failed material takes a form similar to that of the intact material, where the failed strength (at a strain rate of $\dot{\varepsilon}=1 \mathrm{~s}^{-1}$ ) varies linearly between zero at zero pressure and a strength of $\sigma_{f}$ at a pressure of $P_{f}$. At pressures greater than $P_{f}$, the failed strength increases with pressure up to a maximum strength of $\sigma_{\max }^{f}$ according to the following equation;

$\sigma=\sigma_{f}+\left(\sigma_{\max }^{f}-\sigma_{f}\right)\left\{1-\exp \left[-\alpha_{f}\left(P-P_{f}\right)\right]\right\}$,

where

$\alpha_{f}=\sigma_{f} /\left[P_{f}\left(\sigma_{\max }^{f}-\sigma_{f}\right)\right]$.

The reduced effective strength of fractured material under compression (and shear) is thus modeled by Eq. (3). The primary difference between the intact and failed strength is related to the behavior under tension. Intact material can resist plastic deformation under tensile pressures, while failed material has no strength under hydrostatic tension.

Strain rate-dependency is also included in the SWRIG model for both intact and failed material by scaling the strengths defined at $\dot{\varepsilon}=1 \mathrm{~s}^{-1}$ by

$\left(1+C \ln \dot{\varepsilon}^{*}\right)$,

where $C$ is a material constant and $\dot{\varepsilon}^{*}$ is the normalized strain rate, defined as the current strain rate divided by a reference strain rate of $1 \mathrm{~s}^{-1}$. Thus, Eq. (5) characterizes rate effects as the ratio of the strength at the current rate normalized by the strength at the reference rate.

Thermal softening of the strength (intact and failed) is also possible, where the above described strengths can be scaled by a thermal softening factor given by:

$\left(1-\bar{T}^{M}\right)$.

In Eq. (6), $M$ is a material constant and $\bar{T}$ is the homologous temperature given by;

$\bar{T}=\left(\right.$ Temp $\left.-T_{r}\right) /\left(T_{m l t}-T_{r}\right)$,

where Temp is the current temperature, $T_{r}$ and $T_{m l t}$ are the room (ambient) and melting temperatures, respectively. 
Additionally, a dependence on the third invariant $\left(J_{3}\right)$ is possible, which has the effect of reducing the strength for stress states in the tensile meridian. The third invariant dependence is applied to both intact and failed strengths defined above in Eqs. (1)-(4), which are assumed to correspond to stress states on the compressive meridian. Although this feature was not utilized for the current work, the interested reader may find further details regarding this capability in [1].

\section{Damage}

Damage $(D)$ within the SWRIG model evolves according to the accumulation of plastic strain relative to the instantaneous failure strain:

$D=\Sigma\left(\Delta \varepsilon_{p} / \varepsilon_{p}^{f}\right)$

where $\Delta \varepsilon_{p}$ is the current increment of equivalent plastic strain and $\varepsilon_{p}^{f}$ is the current plastic strain-to-failure defined as

$\varepsilon_{p}^{f}=D_{1}\left(P^{*}+T^{*}\right)^{N}\left(1+C_{f} \ln \dot{\varepsilon}^{*}\right)$.

In Eq. (9), $P^{*}=P / \sigma_{\max }$ and $T^{*}=T / \sigma_{\max }$, where $P$ is the current pressure and $T$ is the intact tensile strength of the reference material defined previously. The material constants $D_{1}$ and $N$ control the pressure dependence on the failure strain while $C_{f}$ controls the rate dependence analogous to the constant $C$ with the strength curves.

If failure is predicted to occur $(D=1)$, the material strength is reduced from that described by the intact curve to that of the failed curve (see Fig. 1). The transition from intact to failed strength is controlled by the choice of damage softening or time-dependent failure. Damage softening reduces the strength according to a user-specified constant related to the level of damage at which the onset of softening begins. Time-dependent failure alternatively reduces the strength over a user-specified time.

\section{Equation of State}

The SWRIG model utilizes a pressure versus volumetric strain $\left(\mu=\rho / \rho_{0}-1\right.$, where $\rho$ and $\rho_{0}$ are current and initial densities) relationship that accounts for the possibility of permanent densification (increase in density) under high pressures and bulking (increase in volume) that occurs when glass fails and comminutes. In the model, permanent densification is assumed to begin when $\mu>\mu_{\text {elastic }}$ and is complete when $\mu=\mu_{\text {lock }}$. If no damage has occurred, the pressure under loading is given by,

$P=K_{1} \mu+K_{2} \mu^{2}+K_{3} \mu^{3}$ for $\mu<\mu_{\text {lock }}$ and

$P=P_{\text {lock }}+K_{4}\left(\mu-\mu_{\text {lock }}\right)$ for $\mu>\mu_{\text {lock }}$.
The unloading path depends on the magnitude of permanent densification and the maximum volumetric strain reached, $\mu_{\max }$. Unloading prior to any permanent densification $\left(\mu_{\max }<\mu_{\text {elastic }}\right)$ follows the path given by Eq. (10), hence the response is elastic. After densification is complete $\left(\mu_{\max } \geq \mu_{\text {lock }}\right)$, unloading follows the fully densified path that results in permanent densification of $\mu_{\text {perm }}$ at complete unloading. This fully densified path is determined by interpolation between the paths given by Eqs. (10) and (11) when extrapolated down to zero pressure. Unloading following incomplete densification $\left(\mu_{\text {elastic }}<\mu_{\max }<\mu_{\text {lock }}\right)$ is along a path interpolated between that of Eq. (10) and the completely densified path according to the fraction of densification attained. It is noted that this behavior is almost entirely mechanical; aside from bulking energy discussed below, it contains no temperature or energy dependence as traditional shock EOSs do.

Additional glass behavior handled by the EOS is the dilatancy (bulking), which occurs upon failure. This effect is modeled during the progression of failure (damage) by adding an additional pressure increment to Eqs. (10) and (11) determined from the internal deviatoric energy lost as the strength is reduced from intact to failed. The fraction of this energy loss that is converted to hydrostatic potential energy (bulking) is $0 \leq \beta \leq 1$, which is specified as an input to the model.

\section{Tensile Fracture}

The capability to model location dependent tensile strength and its effect on fracture is a key feature of the SWRIG model. Properly including this behavior requires special consideration in terms of implementation within $\mathrm{CTH}$, therefore a discussion and demonstration of this feature is provided here. Fracture in this context is distinct from other possible types of failure. It corresponds to the creation of new free surfaces associated with a crack and not simply a reduction (or elimination) of strength. This is a complicated phenomenon to handle computationally, as such its treatment tends to be very code specific. For example, some Lagrangian codes handle fracture by erosion (deletion) of elements to create new crack surfaces. This approach may have some adverse properties unless handled with care, and is not applicable to an Eulerian framework. Alternatively, the EPIC Lagrangian code has the capability to handle fracture by converting finite elements to meshless particles. In contrast to both of these approaches, fracture is modeled within $\mathrm{CTH}$ by the insertion of void volume within a cell (for which fracture was detected).

Void insertion increases the pressure of the material (reduces its volume) in the cell to a less tensile state. Some advantages with this approach, aside from compatibility 
with the Eulerian framework, are that it does not violate conservation laws and allows for the possibility of fracture by void growth and fragmentation. Note that this is the standard fracture treatment in $\mathrm{CTH}$ and is not specific to only the SWRIG model.

The approach taken in the implementation of the SWRIG model was to provide a communication mechanism between the model and the standard CTH fracture treatment. This coupling mechanism is the threshold value used in the criterion to detect fracture, triggering void insertion. The maximum hydrostatic tension strengths are used to define this threshold, depending on the condition and location of the material within a cell. The condition (intact or failed) and location (adjacent to failed material, surface, or interior) of glass material within a cell can vary in space and time during the course of a computation, and so the fracture thresholds vary likewise. The fracture threshold of intact material is set as $T, T_{\text {surf }}$, or $T_{\text {int }}$ depending on the appropriate strength location as described above in "Strength Model" section. Failed material has no ability to support tension according to its strength description [see Eq. (3)] and so its fracture threshold is zero.

The use of the hydrostatic tension strengths in this manner is consistent with Holmquist and Johnson's [1] definition and provides the correct tensile fracture behavior according to their original formulation. This is most easily demonstrated by considering the spall plate impact configuration described in [1]. This setup consists of a glass flyer plate impacting a glass target at a velocity of $200 \mathrm{~m} / \mathrm{s}$. Setup dimensions and the generic glass model parameters used here are identical to those provided in [1]. Following initial shock compression, subsequent wave reflections and interactions produce a tensile stress within the target, thereby probing the tensile strength of interior material. The generic glass model parameters used correspond to hydrostatic tension strengths of $T=T_{\text {surf }}=100 \mathrm{MPa}$ and $T_{i n t}=790 \mathrm{MPa}$. Computational results for multiple values of the failure time, $t_{\text {fail }}$, are also shown in Fig. 2 to demonstrate its effect on spall fracture.

The loading sequence of target material is as follows. The initial impact produces elastic longitudinal compression, where subsequent wave interactions unload interior material to zero stress and then load along the same uniaxial strain elastic load path in the tensile portion of pressure space. The material strength in this region is defined by the linear portion of the strength curve $\left(P<P_{i}\right.$, see Fig. 1). Once the stress state along this path reaches the interior strength, plastic strain is generated as a result of yielding. The material at this state is inelastic, while its condition is intact, and failure (and the associated loss of strength) has not yet occurred. The stress state at this intersection consists of equal pressure and interior strength

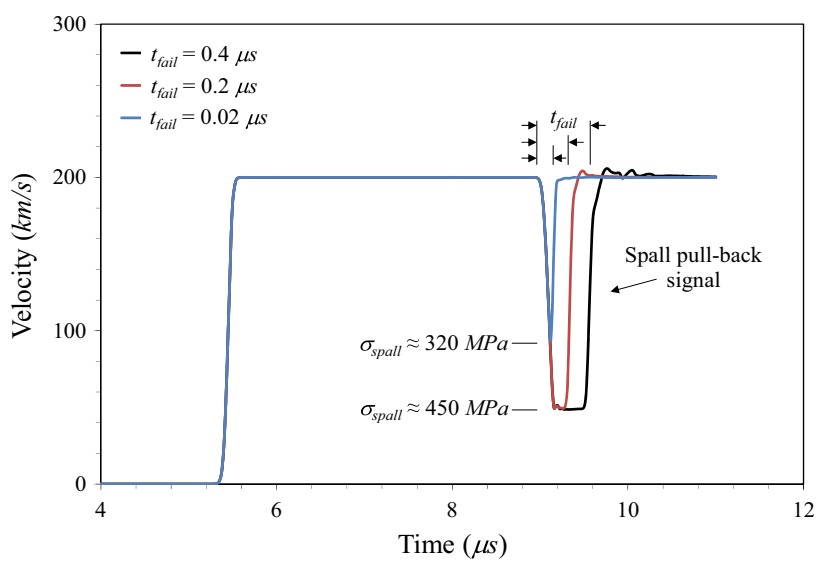

Fig. 2 Spall computation results demonstrating SWRIG model functionality in CTH

contributions of approximately $270 \mathrm{MPa}$, corresponding to a longitudinal tensile (spall) stress of approximately $450 \mathrm{MPa}$. As the yield surface is just reached, the stress state is still elastic and tensile fracture is not yet triggered since this tensile pressure is significantly lower than $T_{i n t}$. The strain to failure in the damage model [Eq. (9)] is zero for any tensile pressures larger than $T=100 \mathrm{MPa}$; therefore, any plastic strain immediately triggers the onset of failure $(D=1)$. Once the onset of failure is triggered, the material strength is gradually reduced from intact to failed material over a time duration of $t_{\text {fail }}$. Note the material is not considered to be (fully) failed until this strength transition is complete. Once complete, the strength of any adjacent intact material is also reduced to that of reference material. At this point, the material is completely failed but has not yet fractured (no void insertion). The fracture threshold for failed material is zero, hence void is inserted under any amount of hydrostatic tension. Therefore, continued tensile loading of this failed material exceeds the current fracture threshold (zero), and the standard CTH fracture model inserts void volume causing fracture.

Results from spall experiments typically consist of the velocity profile recorded at the target rear surface. Velocity profiles from the example spall computations are shown in Fig. 2. The ring-up and plateau to $200 \mathrm{~m} / \mathrm{s}$ corresponds to the initial elastic compression. Unloading into tension is seen as the drop in velocity at approximately $9 \mu$ s. Spall fracture is indicated in these profiles by the pull-back signal produced when the target fractures into two pieces (creating new free surfaces) at the interior location of peak longitudinal tensile stress that was sustained by the material. The height of the pull-back is proportional to the magnitude of the spall strength. The pull-back signal height for $t_{\text {fail }}=0.4 \mu$ s indicates the expected spall strength of $450 \mathrm{MPa}$ determined from the material properties, while the width of the pull-back corresponds to the specified 
Table 1 Compilation of elastic properties of soda-lime glass

\begin{tabular}{llllllll}
\hline Sources & $\rho_{0}\left(\mathrm{~g} / \mathrm{cm}^{3}\right)$ & $c_{L}(\mathrm{~km} / \mathrm{s})$ & $c_{S}(\mathrm{~km} / \mathrm{s})$ & $K(\mathrm{GPa})$ & $G(\mathrm{GPa})$ & $v$ & $\mathrm{HEL}(\mathrm{GPa})$ \\
\hline Bless and Brar (USA) [13] & 2.491 & 5.82 & 3.45 & 45.4 & 26.8 & 0.228 \\
Bless and Brar (German) [13] & 2.53 & 5.83 & 3.47 & 45.3 & 30.4 & 0.226 \\
Willmott and Radford [14] & 2.49 & $5.43^{\mathrm{a}}$ & 3.22 & $45.3^{\mathrm{c}}$ & 29.8 & 0.23 \\
Alexander et al. [21] & 2.5 & 5.761 & 3.437 & $43.7^{\mathrm{c}}$ & $29.5^{\mathrm{c}}$ & 0.224 & 6.0 \\
Holmquist et al. [19, 20] & 2.53 & 5.828 & 3.468 & 45.4 & 30.4 & 0.23 & 5.95 \\
\hline
\end{tabular}

a This wave speed corresponds to 1D (uniaxial) stress

b This value corresponds to the pseudo-elastic limit

c These values were computed from the other properties rather than being measured directly

failure time which delays failure. The expected spall strength is also indicated by the height of the pull-back for $t_{\text {fail }}=0.2 \mu \mathrm{s}$, but the reduced failure time causes the duration of the pull-back to be shorter as the failure transition occurs faster. A reduced spall strength is indicated for the $t_{\text {fail }}=0.02 \mu \mathrm{s}$ pull-back because the very short failure time duration prevents the pull-back from being maintained by the time it reaches the target rear surface. Comparing the CTH computed profiles in Fig. 2 with the sample results originally provided by Holmquist and Johnson [1] show nearly identical profiles, thus demonstrating correct CTH implementation of location-dependent strength, tensile fracture, and time-dependent failure features of the SWRIG model.

The current implementation of the SWRIG model within CTH also handles fracture under pure triaxial tension. Pure triaxial loading produces zero deviatoric stresses, thus failure cannot be triggered by damage accumulation since no plastic strain is produced. In this case tensile fracture would be triggered once the hydrostatic tension exceeded the corresponding fracture threshold. Without this tensile fracture, the material would continue to expand unrealistically, eventually attaining very low-density thermodynamic states, leading to likely numerical instabilities.

\section{Soda-Lime Glass Model Parameters}

A critical component to any predictive simulation is the parameterization of required constitutive models, ideally based on experimental data. To the author's knowledge, soda-lime glass parameters for the SWRIG model have not been previously published in the open literature. A variety of experimental data was used as the basis for SWRIG model parameter development for soda-lime glass. The approach used was similar to that of Holmquist and Johnson [18] in their development for borosilicate glass and draws on data in the open literature covering a broad range of test techniques and loading conditions. While it was desired to estimate the model parameters solely from experimental data, supporting data was not always available. Therefore, an engineering approximation was necessary for the development of some material parameters, which is discussed were applicable.

The soda-lime glass parameter set provided here was developed first before any simulations were performed and were never modified later to improve predicted results. This "blind" approach was taken to present the material model results in a truly predictive manner. A compilation of the experimental data and the procedure used to arrive at the current SWRIG model parameters for soda-lime glass is presented in what follows.

A compilation of elastic properties of soda-lime glass are presented in Table 1. As shown in Table 1, the elastic properties from each of the references are in good agreement with one another. For the SWRIG material parameter set used herein, the values given by Holmquist et al. [19, 20] for the initial density $\left(\rho_{0}\right)$, shear modulus $(G)$, and the initial linear bulk modulus $\left(K_{1}=K\right)$ are used. Other properties shown for reference are the longitudinal $\left(c_{L}\right)$ and shear $\left(c_{S}\right)$ elastic wave velocities, Poisson's ratio $(v)$, and the HEL.

\section{Strength and Damage Properties}

The next step in the SWRIG model development for sodalime glass was to determine the parameters for each of the functional forms describing the strength and damage. The intact strength was first developed as this foundational aspect of the model includes parameters used elsewhere in the model. Parameters describing the failed strength are then determined to complete the strength model development. Finally, fitting of the damage model is presented at the end of this section.

Experimental data describing the intact strength of sodalime was compiled from a variety of references using different types of tests. This collection was used to calculate the measured strength (equivalent stress) and is plotted as a function of pressure in Fig. 3. The inset of this figure shows the data near the zero pressure intercept. For reference, the gray dashed and dashed-dot lines indicate the uniaxial (1D) 
Fig. 3 Soda-lime intact strength versus pressure test data and SWRIG model fit

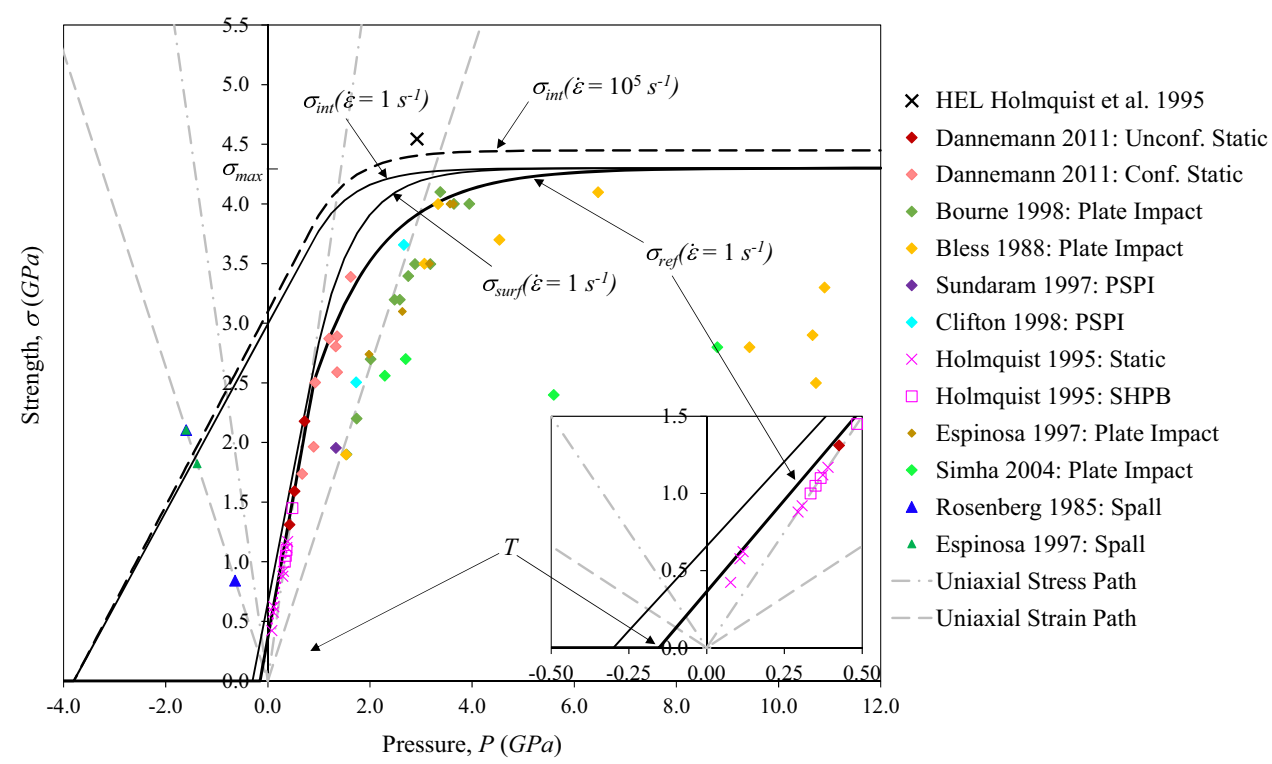

stress and (1D) strain trajectories for elastic response, respectively.

The reader is referred to each publication individually for a complete description of the experimental techniques used to obtain the data in Fig. 3. Here a brief description of each body of work and associated data is provided.

- Dannemann et al. [22] preformed quasi-static compression testing of unconfined (uniaxial stress) and confined (using hydraulic confinement) intact soda-lime (Starphire ${ }^{\circledR}$ ).

- Holmquist et al. [19] performed quasi-static and moderate rate uniaxial compression testing of sodalime float glass at strain rates of $0.001 \mathrm{~s}^{-1}$ (quasi-static) and $250 \mathrm{~s}^{-1}$ (split-Hopkinson pressure bar), in addition to quasi-static tension tests using the "Brazilian" configuration. These tests provide data on the intact strength in both the tensile and compressive regimes.

- Sundaram and Clifton [23] performed pressure-shear plate impact (PSPI) experiments using a very thin layer of soda-lime sandwiched between two hard steel plates. Data from these PSPI tests where no loss of shear strength was observed corresponds to intact material, which is included in Fig. 3.

- Clifton et al. [24] performed PSPI experiments on thick soda-lime specimens. Data from Clifton et al. shown in Fig. 3 corresponds to tests where no loss of shear strength/resistance was observed, thus characterizing intact material.

- Espinosa et al. [25] measured the longitudinal and lateral stress profiles during plate impact experiments on soda-lime. These tests were intended to study shear strength during shock compression, where measurement of lateral and longitudinal stresses provides a direct measure of the strength. Strength data recorded before the failure wave during shock compression is shown in Fig. 3.

- Lateral and longitudinal stress profiles were also measured during plate impact tests performed by Simha and Gupta [26], Bourne et al. [27], and Bless et al. [28], where strength recorded for intact material ahead of the failure wave is shown.

- Rosenberg et al. [29] and Espinosa et al. [25] measured the spall strength of soda-lime using Manganin stress gauges during plate impact (release wave) experiments. These experiments resulted in a spall plane located ahead of the failure wave such that the measured strength was assumed to correspond to intact material.

Determination of a clear HEL for soda-lime glass is complicated due to ramp loading, a pseudo-elastic response, and a complete loss of shear resistance, as observed by Alexander et al. [21]. Time scale, dimensional, and thus strain rate dependencies further complicate the clear determination of an HEL in glasses. These characteristics are likely the source of the large spread in HEL shown in Table 1 and found throughout the literature. The HEL value of $\sigma_{x}=5.95 \mathrm{GPa}$ given by Holmquist et al. is close to the average of the elastic and pseudo-elastic limits (3.5 and 7.5 GPa, respectively) discussed by Alexander et al. This value corresponds to a net longitudinal stress which includes both hydrostatic and strength contributions. For the uniaxial strain load condition under which the HEL was measured, these contributions are related according to $\sigma_{x}=P+2 \sigma / 3$,

where $P$ and $\sigma$ correspond to the pressure and strength at the HEL. The strength and pressure at the HEL determined 
from this relationship using the data from Holmquist et al. [19] is shown in Fig. 3 using an " $X$ " symbol.

Using the collected data, the intact strength of the reference material was estimated and is indicated as $\sigma_{\text {ref }}$ at $\dot{\varepsilon}=1.0 \mathrm{~s}^{-1}$ in Fig. 3. This reference curve was estimated by first determining the maximum hydrostatic tensile strength for intact reference material $(T)$ by extrapolating a linear curve fit of the static test data from Holmquist et al. [19] to zero strength. The resulting tensile strength value of $0.153 \mathrm{GPa}$ was nearly identical to the average of the tensile strengths measured for the Brazilian tests by Holmquist et al. The maximum strength for reference material is identical to that for surface and interior material according to the model (see Fig. $1, P \gg P_{i}$ ); this upper limit is given by a single parameter $\left(\sigma_{\max }\right)$ in the model. Therefore, the maximum strength $\sigma_{\max }=4.3 \mathrm{GPa}$ was estimated from all of the compressive data, focusing on the data from plate impact experiments [25, 27, 28] seen in Fig. 3 in particular. The remaining parameters describing the reference intact strength [Eqs. (1) and (2); $P_{i}, \sigma_{i}$ ] were estimated to follow the various data along the uniaxial stress path and the confined static data from Dannemann et al. [22], as these experiments corresponded to the lowest available strength data for intact material.

It is noted that the maximum strength determined in this way does not agree with the data from Bless et al. [28] at higher pressures. Experimental data points at pressures below approximately $3 \mathrm{GPa}$ generally follow the uniaxial strain elastic load path as one would expect for data below the HEL. Data from Bless et al. above this pressure corresponds to peak Hugoniot stress states above the HEL. It is therefore possible that some damage and/or failure may have occurred at these states as inelastic deformation is expected to have occurred. These samples were initially intact, but it is unclear if these stress states were measured in intact or failed material. Data above the HEL but below pressures of approximately $7 \mathrm{GPa}$ are seen to indicate significant strength, thus these points are considered to be representative of intact material and so were considered in estimating $\sigma_{\max }$. The data at higher pressures (above $8 \mathrm{GPa}$ ) show a considerably lower strength as compared to the data at lower pressures, therefore there is an increased likelihood that these stress states corresponded to failed material.

This interpretation is further supported by the observation that the strength at these high pressures appears to approach the failed strength indicated in measurements behind the failure wave shown in Fig. 4 discussed later. Although the model provides separate parameterizations for the strength of fully intact and failed glass, the progression of failure and associated softening as the strength transitions from intact to failed is intended to provide for this apparent strength degradation that is evident in the data from Bless et al. [28].
The estimated intact strength of surface material is shown in Fig. 3 indicated as $\sigma_{\text {surf }}$ at $\dot{\varepsilon}=1.0 \mathrm{~s}^{-1}$. Wereszczak et al. [30, 31] performed static equibiaxial flexure (ring-on-ring) and Hertzian ring crack initiation tests of a soda-lime-silicate float glass $\left(\right.$ Starphire $\left.^{\circledR}\right)$. These experiments measured the tensile failure stress for each of the tinside and air-side surfaces of the specimens. This data was used to roughly estimate the tensile strength of intact surface material $\left(T_{\text {surf }}\right)$ as approximately $0.3 \mathrm{GPa}$, with the value of $D_{\text {surf }}$ determined following the relationships shown in Fig. 1.

The intact interior strength of soda-lime glass is indicated as $\sigma_{\text {int }}$ in Fig. 3 at $\dot{\varepsilon}=1.0 \mathrm{~s}^{-1}$. This curve was estimated using the experimental data of Rosenberg et al. [29] and Espinosa et al. [25] who found that the spall strength of soda-lime glass exceeded $3 \mathrm{GPa}$ for intact material. The tensile strength of interior material $\left(T_{i n t}\right)$ was selected such that the resulting interior strength curve in the hydrostatic tensile regime nominally follows/envelopes spall strength data from Rosenberg et al. and Espinosa et al. The chosen value of $T_{\text {int }}=3.8 \mathrm{GPa}$, yields a reasonable representation of this spall data in strength-pressure space, corresponding to a spall strength (net longitudinal stress) of approximately $3 \mathrm{GPa}$. The corresponding value of $D_{\text {int }}$ was then determined from the relationships shown in Fig. 1. This parameterization is regarded as a reasonable first estimate given the limited data currently available. It is acknowledged that this part of the model parameterization is one of the most uncertain due to the general lack of supporting experimental data.

Some of the tests performed by Holmquist et al. [19] included identical loading conditions performed at different rates, these provided a direct dataset to determine the strain rate effect on the strength $(C)$. Holmquist et al. used this soda-lime data with the JH-2 model [19] to characterize the observed rate-dependence. Since the form of the rate-dependency in the JH-2 and SWRIG models are identical, the value for $C$ originally determined by Holmquist et al. was used directly here. This estimated ratedependence is illustrated with the interior strength curves in Fig. 3 at $\dot{\varepsilon}=1 \mathrm{~s}^{-1}$ (solid curve) and $\dot{\varepsilon}=10^{5} \mathrm{~s}^{-1}$ (dashed curve). Consistency of the maximum strength represented by the SWRIG model at the nominal plate impact strain rate of $\dot{\varepsilon}=10^{5} \mathrm{~s}^{-1}$ with the measured strength at the HEL (shown as the "X") is seen in Fig. 3.

Parameter development describing the failed strength is similar to that outlined above for intact material. Experimental measurements on the strength of failed soda-lime were compiled from the literature and are shown in Fig. 4 as a function of pressure. Brief descriptions of each work providing failed strength data are given here. The reader is referred to each publication individually for a complete description of the experiments. 
Fig. 4 Soda-lime failed strength versus pressure test data and SWRIG model (Color figure online)

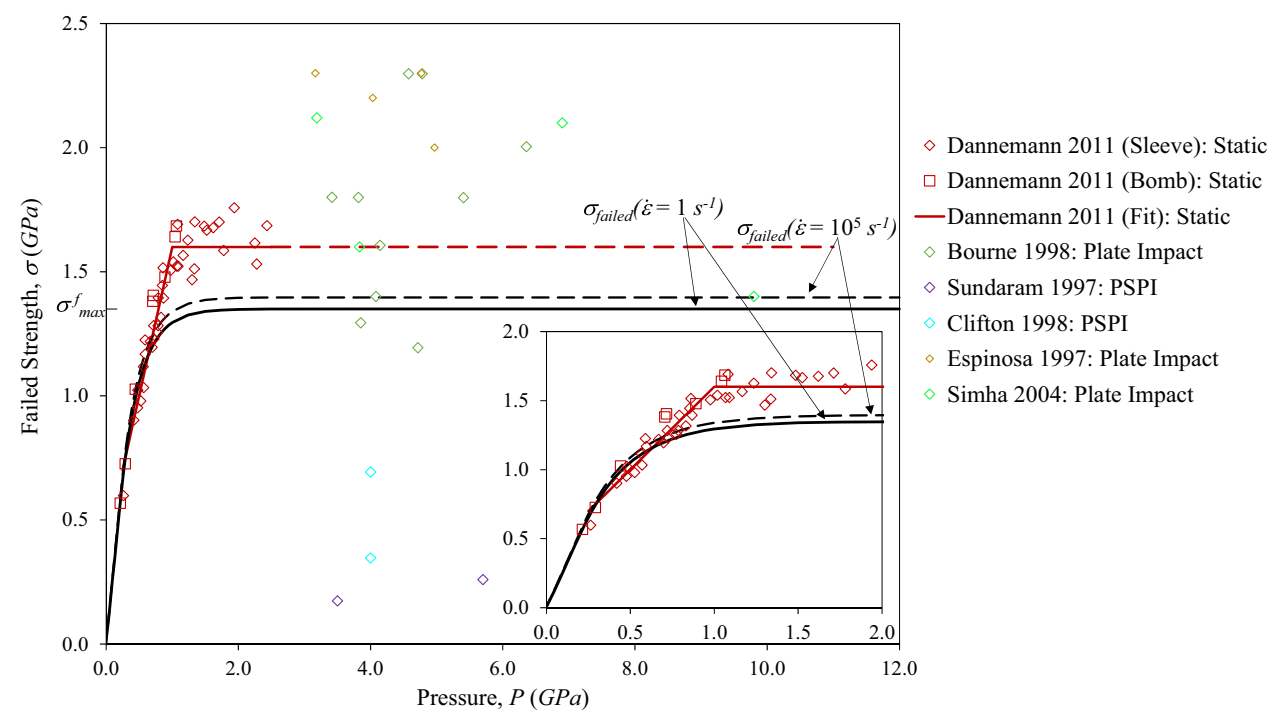

- In addition to data on intact material, Dannemann et al. [22] also presented data from quasi-static confined compression tests on pre-damaged (from a thermal shock technique) soda-lime (Starphire ${ }^{\circledR}$ ) using both mechanical (sleeve) and hydraulic (bomb) confinement. A simple bilinear curve fit to this data provided by Dannemann et al. is also shown in Fig. 4, where the dashed red line segment corresponds to extrapolation of this fit to high pressure.

- Lateral and longitudinal stresses measured behind the failure wave during the plate impact experiments by Bourne et al. [27], Simha and Gupta [26], and Espinosa et al. [25] provided data on the strength of damaged/failed soda-lime during shock compression.

- Data from the PSPI experiments by Clifton et al. [24] and Sundaram and Clifton [23], where a loss of shearing resistance was observed, provides additional measurements on the strength of damaged/failed glass under large shear strains and strain rates simultaneously at high pressures.

Using the data in Fig. 4, the failed material strength curve was determined by first estimating the maximum failed strength $\left(\sigma_{\max }^{f}\right)$ from the failed material test data (static, plate impact, and PSPI) in the high pressure plateau region. The maximum strength indicated by the plate impact, PSPI, and static data was approximately 2, 0.4, and $1.6 \mathrm{GPa}$, respectively. This large spread in the data indicates that the apparent strength of failed glass may be dependent on the mode of deformation as well as the pressure, that is, the failed strength appears to be significantly less under combined pressure-shear as compared to under longitudinal compression alone. The lower failed strength indicated by the PSPI data may also be a consequence of the very large shear strains $(\sim 2.0)$ attained and the possibility of localization occurring under this mode of deformation. These observations complicate the determination of a single maximum (compressive) failed strength value as required by the model, which does not otherwise depend on the state of stress or strain. Therefore all existing data was averaged to provide the best available first estimate from multiple test conditions. This yielded an average maximum failed strength of $1.35 \mathrm{GPa}$. The values for $P_{f}$ and $\sigma_{f}$ [Eqs. (3) and (4)] govern the slope and curvature of the failed strength at lower pressures. These parameters were estimated to reproduce the pressure-sensitivity of the low-pressure (static) data from Dannemann et al. The resulting failed strength of the SWRIG model for soda-lime is shown as the solid black line at a strain rate of $1.0 \mathrm{~s}^{-1}$ in Fig. 4, while the dashed black line shows the corresponding failed strength at $\dot{\varepsilon}=10^{5} \mathrm{~s}^{-1}$. Each of the intact and failed strength curves estimated for soda-lime glass with the SWRIG model are illustrated together in Fig. 5 at strain rates of $\dot{\varepsilon}=1$ and $10^{5} \mathrm{~s}^{-1}$.

For this work, the time-dependent damage softening option was specified and calibrated based on data in the literature which indicated that soda-lime glass demonstrates time-dependent inelastic behavior. This data included the PSPI experiments by Sundaram and Clifton, and Clifton et al., as well as the plate impact experiments by Simha and Gupta, and Espinosa et al. Using this data, the average time to failure under shock loading, $t_{\text {fail }}$, was estimated to be approximately $0.6 \mu$ s.

No experimental data was found to support the remaining features of the SWRIG model. Soda-lime glass data characterizing thermal softening $(M)$ and the effect of the third invariant $\left(J_{3 f a c t}\right)$ was not found; therefore these features were deactivated in the current work. All of the deviatoric internal energy lost upon failure was assumed to 
Fig. 5 Rate dependence of soda-lime strength with the SWRIG model

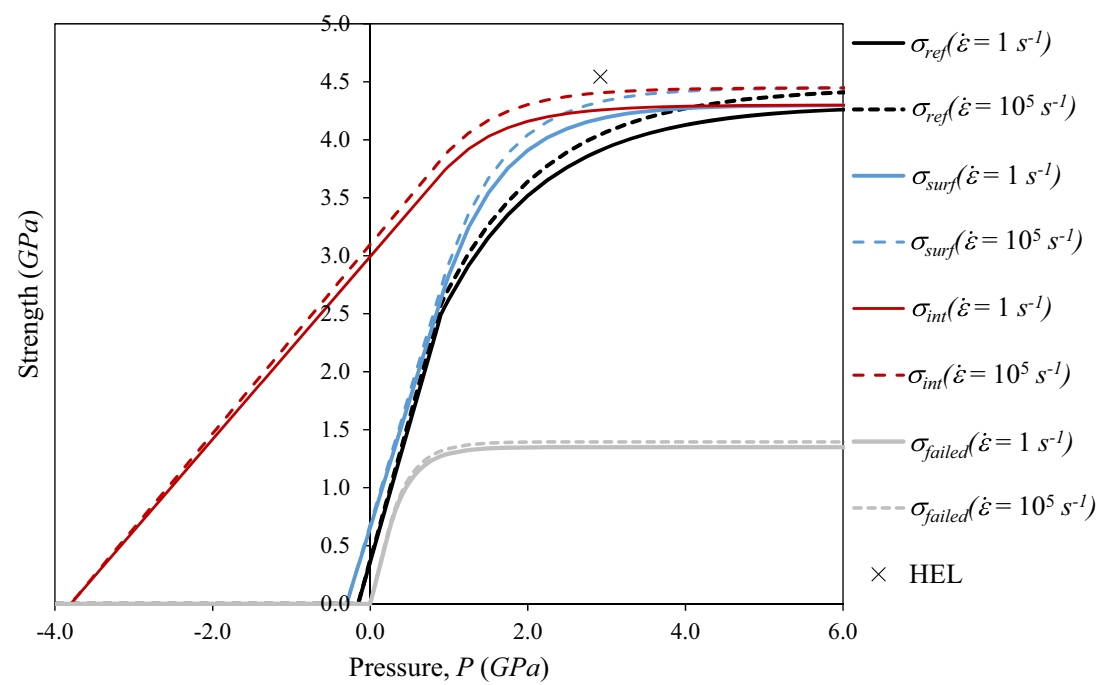

go into bulking $(\beta=1)$. This was chosen as it conserves energy, is consistent with the setting used for soda-lime glass by Holmquist et al. [19] with the JH-2 model, and because there is no direct way to measure this to determine otherwise.

No experimental data was found to directly calibrate the parameters of the failure strain [Eq. (9); $D_{1}, N$, and $C_{f}$ ] used in the SWRIG damage model. Noticing that the form of the failure strain in the SWRIG model is identical to that of the JH-2 model [19], the functional dependence determined previously by Holmquist et al. for soda-lime glass with JH-2 was assumed here. The SWRIG failure strain parameters were thus determined to recover this assumed functional dependence. This is regarded as a reasonable first estimate give the lack of data; it is acknowledged that the parameterization of this model feature is one of the less well established and potentially requires some refinement.

\section{Equation of State Properties}

The volumetric response of soda-lime glass with the SWRIG model EOS is developed primarily from Hugoniot data obtained from plate impact experiments by Alexander et al. [15], Grady and Chhabildas [32], Dandekar [33], and Dandekar and Beaulieu [34]. This collection of data is shown in Fig. 6 and corresponds to measurements of longitudinal stress versus compressive volumetric strain. The inset of this figure shows the lower-pressure data at a reduced scale to more easily view lower-pressure data near the HEL. The hydrostatic pressure was estimated from longitudinal stress measurements below the HEL, where the deviatoric stress contribution could be easily computed and subtracted from the longitudinal stress using Eq. (12). These estimated pressure-compression data points are shown as open black diamonds in Fig. 6. As the stress increases, well above the HEL in particular, strength effects become less pronounced and thus the Hugoniot data is assumed to be a reasonable representation of the hydrostat.

In studying this experimental data it was observed that the hydrostat of soda-lime glass determined previously by Holmquist et al. with the JH-2 model was a good fit to this data up to approximately $20 \mathrm{GPa}$. Since the form of the JH2 hydrostat is identical to that of the SWRIG model when permanent densification is neglected [Eq. (10): $K_{1}, K_{2}, K_{3}$ ], the values from Holmquist et al. were used directly here. Extrapolation of Eq. (10) above $20 \mathrm{GPa}$ results in an overly stiff response as compared to the high pressure data. Therefore, SWRIG model parameters related to the completely densified response [Eq. (11); $K_{4}$ and $\mu_{\text {lock }}$ ] were estimated such that the hydrostat followed the test data above this $20 \mathrm{GPa}$ threshold.

A maximum permanent densification of $6.3 \%$ was measured by Ji et al. [35] for soda-lime glass after complete unloading from static compression to approximately $25 \mathrm{GPa}$. These measurements showed a saturation in permanent densification above a pressure of approximately 20 GPa. No additional experimental data was found for sodalime at pressures higher than those tested in this work. Therefore, the value at saturation measured by Ji et al. was assumed for the current model. This value was input directly into the model $\left(\mu_{\text {perm }}\right)$ and is shown in Fig. 6 as a thin yellow $\mathrm{X}$ on the horizontal axis. The onset of permanent densification $\left(\mu_{\text {elastic }}\right)$ was assumed to occur at the HEL (shown as a black $\mathrm{X}$ in Fig. 6); consistent with its definition and within the range indicated by Ji's experiments.

The resulting hydrostat of the SWRIG model for sodalime glass is shown in Fig. 6 as a solid black line with arrows indicating the hydrostatic load path $(P)$ for a load- 
Fig. 6 Longitudinal stress and pressure versus compression (strain) for soda-lime glass and SWRIG model (Color figure online)

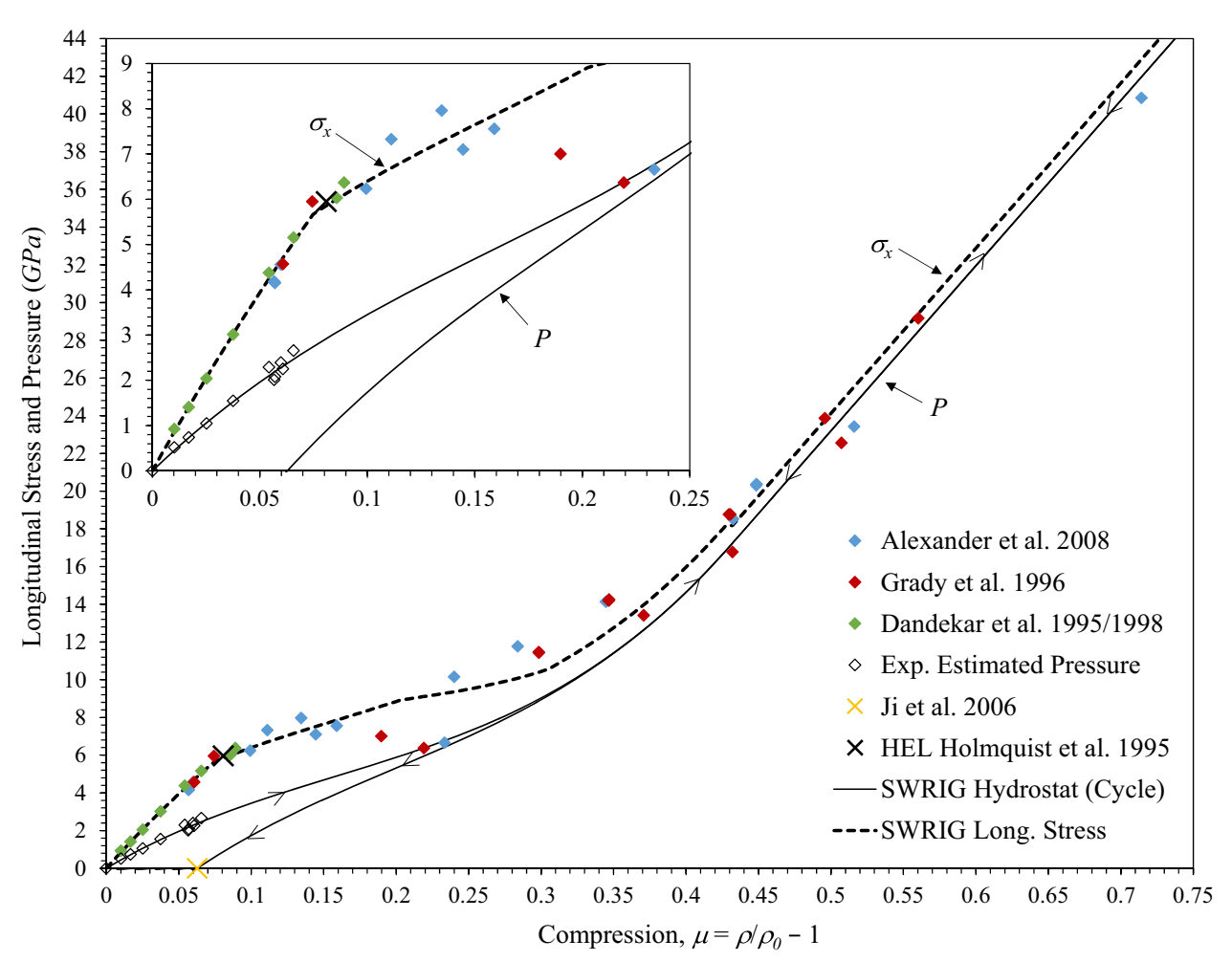

\begin{tabular}{llllllll}
\hline$\rho_{0}$ & $2.53 \mathrm{~g} / \mathrm{cm}^{3}$ & $G$ & $30.4 \mathrm{GPa}$ & $T$ & $0.153 \mathrm{GPa}$ & $\sigma_{f}$ & $0.567 \mathrm{GPa}$ \\
$K_{1}$ & $45.4 \mathrm{GPa}$ & $C_{V}$ & $880 \mathrm{~J} /(\mathrm{K} \mathrm{g})$ & $\sigma_{i}$ & $2.5 \mathrm{GPa}$ & $P_{f}$ & $0.214 \mathrm{GPa}$ \\
$K_{2}$ & $-138 \mathrm{GPa}$ & $T_{r}$ & $298 \mathrm{~K}$ & $P_{i}$ & $0.9 \mathrm{GPa}$ & $\sigma_{\max }^{f}$ & $1.35 \mathrm{GPa}$ \\
$K_{3}$ & $290 \mathrm{GPa}$ & $T_{m l t}$ & $997 \mathrm{~K}$ & $\sigma_{\max }$ & $4.3 \mathrm{GPa}$ & $D_{1}$ & 0.0736 \\
$K_{4}$ & $87.6 \mathrm{GPa}$ & $M$ & 0 & $D_{\text {surf }}$ & 0.976 & $N$ & 0.85 \\
$\mu_{\text {elastic }}$ & 0.081 & $\beta$ & 1.0 & $D_{\text {int }}$ & 0.569 & $C_{f}$ & 0 \\
$\mu_{\text {perm }}$ & 0.0628 & $J 3_{\text {fact }}$ & 0 & $C$ & 0.003 & $t_{\text {fail }}$ & $0.6 \mu \mathrm{s}$ \\
$\mu_{\text {lock }}$ & 0.43 & & & & & & \\
\hline
\end{tabular}

Table 2 SWRIG model parameters for soda-lime glass unload sequence in which complete permanent densification occurs. The predicted hydrostat is seen to follow the pressure estimated from the low pressure Hugoniot data in addition to matching the Hugoniot data points near $6 \mathrm{GPa}$ ( $\mu \approx 0.21$ ), consistent with a complete loss of strength as observed in [21] over a similar stress range. Test data above approximately $12 \mathrm{GPa}$ is also seen to be represented well by the SWRIG model hydrostat consistent with a loss of strength in the material at these pressures.

Also shown in Fig. 6 is the longitudinal stress $\left(\sigma_{x}\right)$ computed from a uniaxial (1D) strain compression load path at $\dot{\varepsilon}=10^{5} \mathrm{~s}^{-1}$. The computed longitudinal stress is seen to match the Hugoniot data well in the elastic region, verifying the assumption of a constant shear modulus. The loss of strength seen in the Hugoniot data just above the HEL to approximately $12 \mathrm{GPa}$ is reasonably represented by the model as well.

\section{Soda-Lime Properties Summary}

The SWRIG model parameters estimated for soda-lime glass are summarized in Table 2. In addition to the parameters detailed in "Strength and Damage Properties" and "Equation of State Properties" sections, the specific heat $\left(C_{V}\right)$ and melting temperature $\left(T_{m l t}\right)$ of soda-lime glass are also required. Values for these constants were taken from a material datasheet for soda-lime-silica float glass [36].

\section{Computational Evaluation}

In this section we compare numerical results from the SWRIG model within CTH to flyer plate and Taylor rod impact experiments on soda-lime glass. These two test 
methods were chosen as they subject the sample material to very different load conditions and stress-deformation states while providing insightful diagnostics on constitutive response. The plate impact experiments were performed by Alexander et al. [15] to study the shock response of sodalime glass under uniaxial strain conditions at high rates and stresses. The Taylor impact experiments were performed by Willmott and Radford [14] to study the deformation and failure of soda-lime glass at lower rates and stresses. In these Taylor tests, the rod specimens are subjected to uniaxial strain compression upon impact, which is quickly released to a state of uniaxial stress thereafter. Simulation of this combination of experiments provides an initial evaluation of the predictive capabilities of the SWRIG model and the suitability of the derived model parameters for soda-lime glass.

\section{Flyer Plate Impacts}

Flyer plate impact experiments conducted on soda-lime glass by Alexander et al. [15] utilized two different configurations as shown schematically in Fig. 7. The plate reverberation configuration in Fig. 7a was used primarily to measure the release response of the glass. The transmitted wave configuration in Fig. 7b was designed to measure shock wave transmission through the glass target. Details of Alexander's experiments considered here are repeated in Table 3. These experiments resulted in peak stresses in the range of approximately 4-41 GPa. Each transmitted wave experiment incorporated a lithium fluoride ( $\mathrm{LiF}$ ) window at the back surface of the glass target. No windows were used in the plate reverberation tests. The velocity of the target rear face was measured during the each experiment using VISAR [37].

Numerical simulations of each configuration listed in Table 3 were carried out using CTH with the SWRIG model and the soda-lime glass parameters in Table 2. The remaining components of each configuration (Lexan, LiF, sapphire, tantalum, and copper) were modeled with EOSs and strength models based on data in [38-40]. The target rear surface velocity was output during each computation at a location corresponding to the VISAR probe points in the experiments.

A comparison between the $\mathrm{CTH}$ simulation results and the test data from the reverberation experiments (SODA2-4) are shown in Fig. 8. For each of these simulations, the number and timing of reverberations, as well as the magnitude of the steady state velocity is predicted reasonably
Fig. 7 Experimental configurations from Alexander et al. [15], a plate reverberation and $\mathbf{b}$ transmitted wave tests

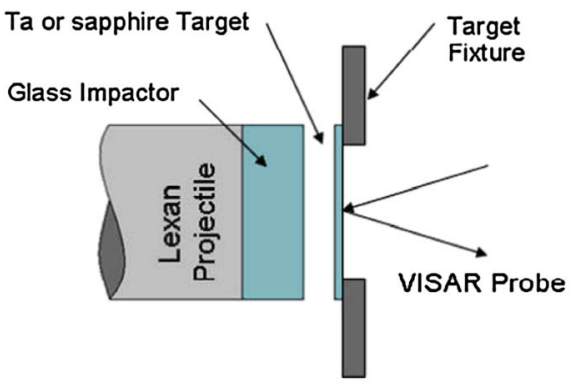

(a)

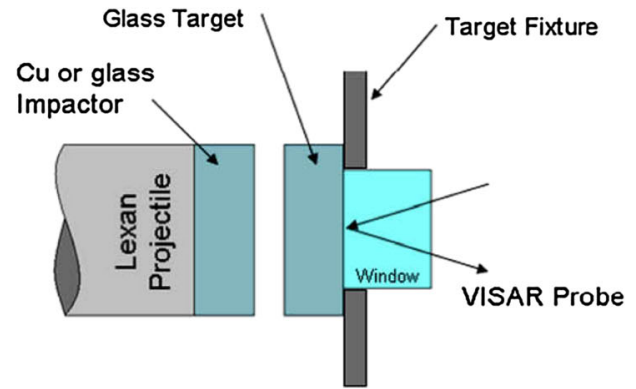

(b)

Table 3 Experimental configurations of plate impact experiments by Alexander et al. [15]

\begin{tabular}{|c|c|c|c|c|c|c|c|}
\hline Configurations & $\begin{array}{l}\text { Shot } \\
\text { names }\end{array}$ & $\begin{array}{l}\text { Impactor } \\
\text { materials }\end{array}$ & $\begin{array}{l}\text { Impactor thickness } \\
(\mathrm{mm})\end{array}$ & $\begin{array}{l}\text { Target } \\
\text { materials }\end{array}$ & $\begin{array}{l}\text { Target thickness } \\
(\mathrm{mm})\end{array}$ & $\begin{array}{l}\text { Impact velocity } \\
(\mathrm{km} / \mathrm{s})\end{array}$ & $\begin{array}{l}\text { Stress } \\
(\mathrm{GPa})\end{array}$ \\
\hline \multirow{3}{*}{$\begin{array}{l}\text { Plate } \\
\text { reverberation }\end{array}$} & SODA-2 & Soda-lime & 24.478 & Sapphire & 0.511 & 0.40 & 4.24 \\
\hline & SODA-3 & Soda-lime & 12.558 & Tantalum & 0.417 & 2.02 & 20.38 \\
\hline & SODA-4 & Soda-lime & 12.588 & Tantalum & 0.422 & 3.22 & 40.94 \\
\hline \multirow{6}{*}{$\begin{array}{l}\text { Transmitted } \\
\text { wave }\end{array}$} & SODA-7 & Copper & 5.021 & Soda-lime & 5.723 & 0.74 & 7.25 \\
\hline & SODA-8 & Copper & 4.893 & Soda-lime & 5.725 & 1.13 & 8.08 \\
\hline & AT-4 & Soda-lime & 6.02 & Soda-lime & 6.00 & 2.38 & 14.25 \\
\hline & AT-5 & Copper & 2.35 & Soda-lime & 5.96 & 1.94 & 18.76 \\
\hline & AT-6 & Copper & 2.38 & Soda-lime & 5.98 & 2.33 & 23.91 \\
\hline & AT-9 & Soda-lime & 5.98 & Soda-lime & 5.97 & 0.64 & 4.62 \\
\hline
\end{tabular}



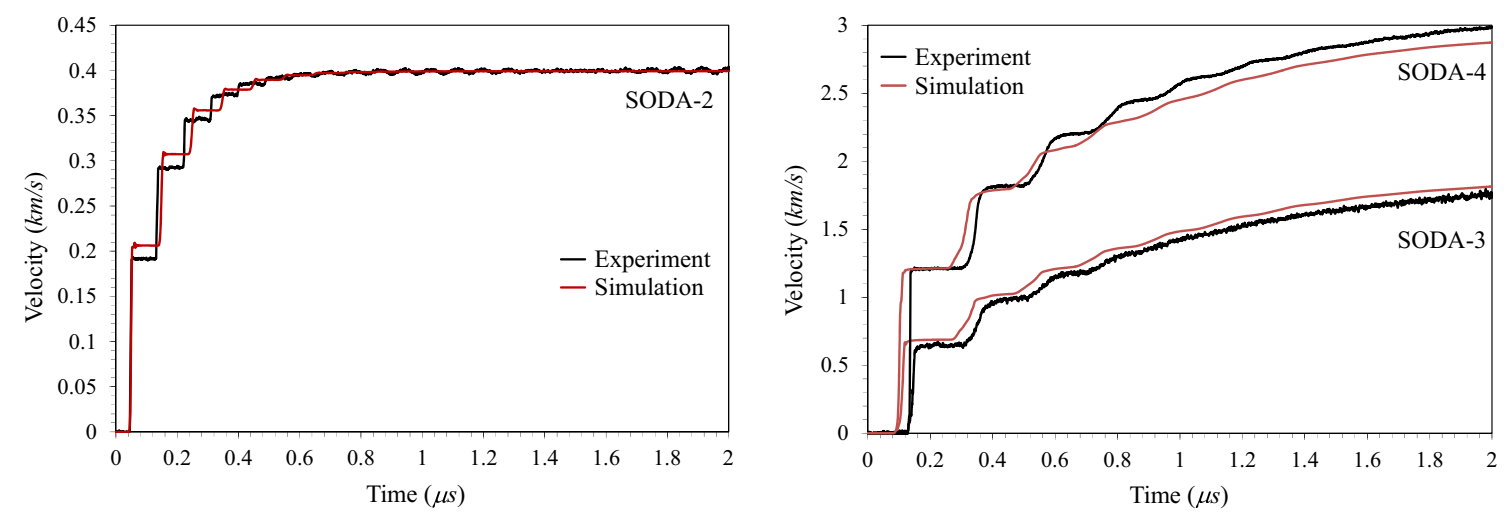

Fig. 8 Comparison of measured [15] and predicted wave profiles for reverberation experiments

well relative to the experimental data. While the timing of each reverberation is predicted reasonably well, all of the numerical results for SODA-3 and SODA-4 appear slightly earlier than the experimental data. This uniform time shift may be due to some systematic differences in the simulated configurations relative to the experiments, such as a discrepancy in the assumed constitutive behavior of tantalum or sapphire. Modification to the pressure-dependence on the glass sound speed at high pressures may also improve the timing correlation. The timing of the SODA-2 prediction starts out nearly identical to the experimental data, but a progressively larger delay in the timing of subsequent reverberations indicates a possible discrepancy in the glass model release at low stress. The predicted step heights of SODA-2 are in reasonable agreement with the measurement, although the relatively small discrepancies seen point to a slight deviation from the experimental release states. Finally, the minor differences in the late-time velocity plateau level for SODA-3 and SODA-4 may indicate slight discrepancies in the way in which permanent densification was modeled, the combined effects of release with damage, or in the assumed constitutive behavior of the target material.

The data measured during the transmitted wave profile experiments performed at lower stress levels (SODA-7, SODA-8, and AT-9) are compared to computational results in Fig. 9. The numerical prediction of the lowest stress shot (AT-9) captures the initial plateau level well but not the slight relaxation seen shortly thereafter. Simulation results of the release portion of this profile matches the experiment in terms of timing and final level, but the release is much more abrupt, possibly due to a relaxation response that is not captured by the computation.

At increasing stress levels, the measured profiles for SODA-7 and SODA-8, respectively show smooth curvature to the plateau levels, which likely indicates time-dependent or more gradual inelastic behavior. The corresponding profiles from the computations instead display a two-wave structure indicative of a more abrupt elastic-plastic transition due to the lack of kinetics in this model. While the transitions are not well captured, the plateau levels are comparable to the measured profiles. SODA-7 again is missing some slight relaxation. The release portion of SODA-7 is reasonably captured by the simulation.

The results for the simulation of SODA-8 differ from the other lower stress experiments. The very gradual rise to the plateau in the experimental data is contrasted by distinct multiple-wave structure in the simulation results. The initial elastic wave in the simulation (up to $\sim 0.35 \mathrm{~km} / \mathrm{s}$ ) matches the experimental data well. The steep front of the second wave in the predicted result is due to the elasticplastic transition, while the dip in the profile immediately following this plastic wave is due to the failure wavefront approaching the rear surface of the glass target (at the LiF window interface). Subsequent smooth curvature to the plateau value predicted shows some similarities to the experimental profile. This smooth transition in the computation is due to time-dependent failure (strength transition from intact to failed) and is a consequence of the glass completely failing near the rear surface. Finally, the predicted peak plateau level matches the experiment well, as does the release.

The simulations of SODA-7 and SODA- 8 exhibit a sharp elastic-plastic transition which occurs at the same stress level and thus indicate a constant, rather than timedependent threshold elastic limit in the model. Examining the SWRIG strength curves for soda-lime glass in Fig. 3, it is clear that the flow stress under these high pressure conditions corresponds to the plateau region $\left(\sigma_{\max }\right.$ at the applied strain rate) and so this threshold is constant according to the model. A more pronounced time-dependence in the strength model is needed to reproduce the smoother transition in the wave profiles observed experimentally. A potential SWRIG model parameter change which could mimic this behavior might be to extend the 

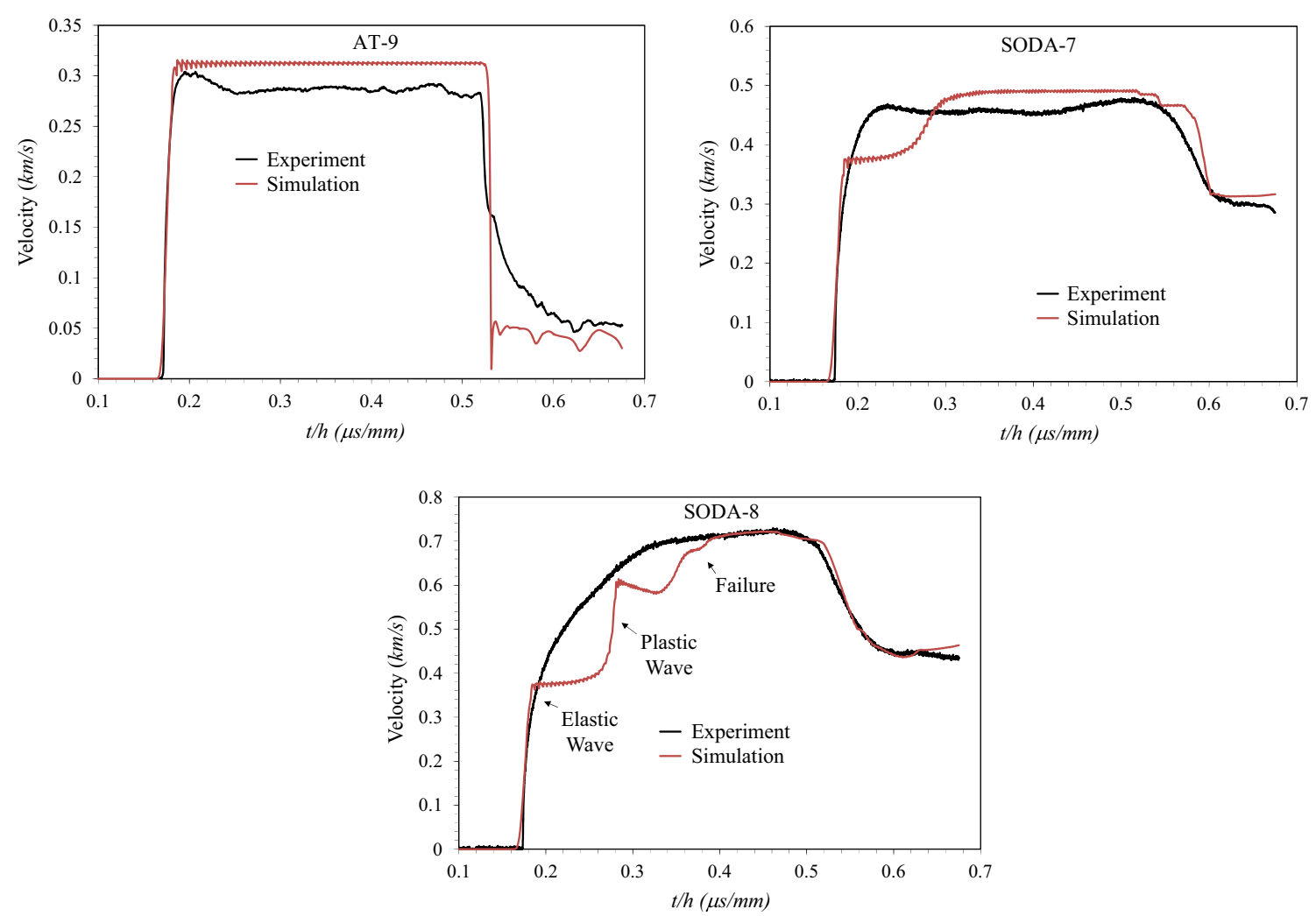

Fig. 9 Comparison of measured [15] and predicted wave profiles for transmitted wave experiments at low-stress

pressure-sensitive (linear) region of the intact strength to higher pressures.

The data measured during the transmitted wave profile experiments performed at higher stress levels (AT-4-6) are compared to results from the computations in Fig. 10. AT4 is shocked to a moderate level such that a more appreciable elastic-plastic wave structure is evident in the measured profile. The experimental profile for this test again illustrates a gradual elastic-plastic transition attributed to transition kinetics, but the computation displays a distinct two-wave structure, as was observed in the previous comparisons for SODA-7 and SODA-8. This is to be expected as transition kinetics are not included in the current model. The timing of the release in AT- 4 is predicted reasonably well. The quasi-elastic release due to strength is more pronounced in the simulation and the bulk release is shallower, but the transition to bulk unloading occurs at approximately the correct time.

Looking at the profile for the next higher shock stress, AT-5, the gradual elastic-plastic response is closer to being overdriven. The computation matches the timing and level of both the elastic and plastic shocks very well. The lack of a smooth transition between the elastic and plastic waves as in the experiment is again a result of the roughly constant (time-independent) strength given by the SWRIG model at high pressures, as described previously. For this configuration, a failure wave is predicted to form shortly after the plastic shock, where the delay (related to the failure time, $\left.t_{\text {fail }}\right)$ of this trailing wave is believed to be the reason for the slightly increased plateau width as compared to the observed profile. The release is captured reasonably in the computation although the predicted signal levels off later (at approximately $0.3 \mu \mathrm{s} / \mathrm{mm}$ ), which may also be a consequence of the failure time.

The elastic response is nearly overdriven at the highest stress shot (AT-6); therefore, the discrepancies in the elastic-plastic transition seen in the lower stress predictions, while still apparent, are less prevalent here. The dispersed (ramped) elastic wavefront and nearly immediate plastic wave are predicted well aside from the modest time shift (ahead) of the prediction relative to the measurement. As seen in the simulation results for AT-4 and AT-5, the width of the plateau at peak shock stress predicted for AT-6 is somewhat larger than in the measured profile. This trend indicates modification to the pressure-dependence on the glass sound speed at high pressures may be needed, which is similar to the conclusion for the reverberation shots (SODA-3 and SODA-4) which were shocked to comparable stress levels. The release in the computation is in fair agreement with the experiment in terms of the release slope, but the predicted result is seen to level off later and maintain a somewhat lower level similar to AT-5. 

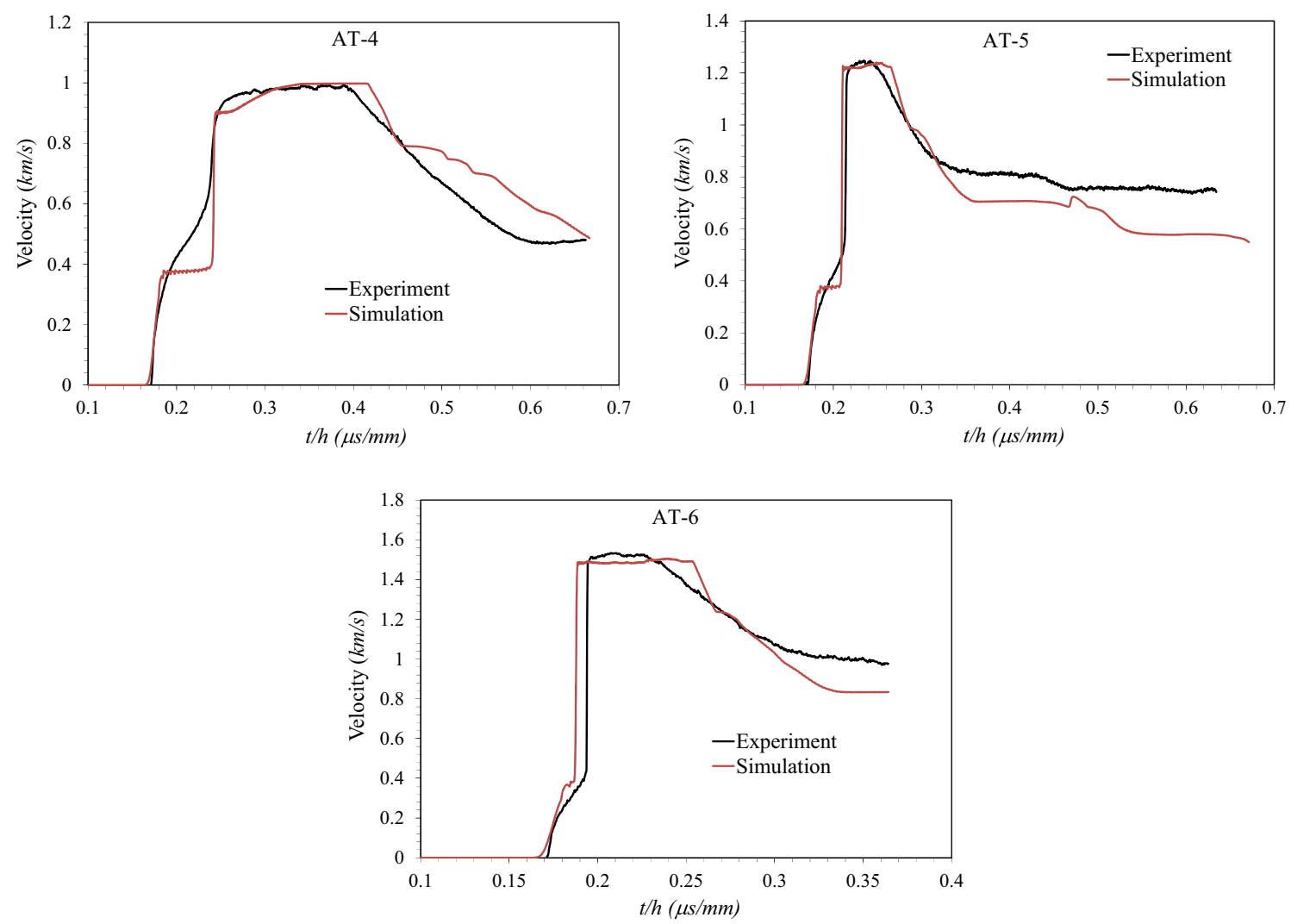

Fig. 10 Comparison of measured [15] and predicted wave profiles for transmitted wave experiments at high-stress

Correlation with the release portion of the profiles for these higher stress configurations (AT-4-6) are not as good as for the lower stress shots (AT-9, SODA-7, and SODA8). This particular discrepancy points to issues with the unloading wave speed upon release from these higher stress states. In the model, permanent densification dictates how the unloading path deviates from the loading path, and thus significantly affects the unloading wave speed. The loading wave speed appears to match the experimental results much better than unloading speed at these stress levels; this suggests some modification to the assumed permanent densification may be needed. Such modifications might also resolve the potential wave speed discrepancies seen in the comparisons with reverberation shots SODA-3 and SODA-4. Recall that the permanent densification model was estimated from limited data corresponding to static compression-decompression experiments. This data simply provided a maximum permanent densification at zero pressure and so did not provide any information on the unloading path traversed.

\section{Taylor Rod Impact}

The Taylor impact experiments by Willmott and Radford [14] studied the dynamic deformation and failure behavior of soda-lime glass rods at moderate impact velocities. In their work, they utilized both classic and symmetric Taylor rod configurations. For the classic configuration, cylindrical glass rods impacted a hardened steel anvil. The symmetric configuration consisted of one glass rod impacting an identical, stationary glass rod. The rods measured $10 \mathrm{~mm}$ in diameter and $100 \mathrm{~mm}$ in length $(L / D=10)$ for all cases. Fourteen experiments were performed in total, covering impact velocities of $161-485 \mathrm{~m} / \mathrm{s}$. A summary of each of the experiments is listed in Table 4.

Willmott and Radford used high-speed photography as the primary diagnostic during their experiments, which allowed for visual identification of deformation and failure during impact. The use of back lighting with the initially transparent (virgin) rods allowed for failed glass to be identified as opaque regions due to cracks within the fractured material which prevented light transmission. Based on the available data, there is no way to know with any certainty whether the failure observed in a given image is located on the rod surface, the interior, or both.

For the work presented herein, attention was focused on specimens SL5 (classic) and SL8 (symmetric) as complete photographic sequences of these impact events were available for direct comparison to simulation results. Predicted failure front velocities were derived from simulation 
Table 4 Summary of Taylor impact experiments by Willmott and Radford [14]

\begin{tabular}{llll}
\hline Specimens & Configurations & Impact velocity $(\mathrm{m} / \mathrm{s})$ & Impact pressure $(\mathrm{GPa})$ \\
\hline SL1 & Classic & $370 \pm 5$ & $3.8 \pm 0.1$ \\
SL2 & Classic & $161 \pm 5$ & $1.7 \pm 0.1$ \\
SL3 & Classic & $370 \pm 5$ & $3.8 \pm 0.1$ \\
SL4 & Classic & $379 \pm 5$ & $3.9 \pm 0.1$ \\
SL5 & Classic & $370 \pm 5$ & $3.8 \pm 0.1$ \\
SL6 & Classic & $391 \pm 5$ & $4.1 \pm 0.1$ \\
SL7a & Symmetric & $379 \pm 5$ & $2.8 \pm 0.1$ \\
SL7b & Symmetric & $379 \pm 5$ & $2.8 \pm 0.1$ \\
SL8 & Symmetric & $391 \pm 5$ & $2.8 \pm 0.1$ \\
SL9 & Symmetric & $485 \pm 1$ & $3.5 \pm 0.1$ \\
SL10 & Symmetric & $309 \pm 5$ & $2.2 \pm 0.1$ \\
SL11 & Symmetric & $274 \pm 5$ & $2.8 \pm 0.1$ \\
SL12 & Symmetric & $330 \pm 5$ & $3.4 \pm 0.1$ \\
SL13 & Symmetric & $313 \pm 5$ & $3.2 \pm 0.1$ \\
\hline
\end{tabular}

results in a manner analogous to the experiments; through visual measurement of the front position through time. Simulations were also performed for each configuration at the highest impact velocity/pressure tested; specimen SL6 (classic) and SL9 (symmetric). For these two specimens, comparison of computational and experimental results was limited to failure front velocities since corresponding high speed imagery was not provided by Willmott and Radford.

All CTH computations were conducted in 3D utilizing two planes of symmetry orthogonal to the impact face. Uniform computational grids of $0.1 \mathrm{~mm}$ cubic cells provided a 50 cell resolution of the rod radius. This mesh resolution was found to resolve gradients and failure propagation well, although complete evaluation of mesh convergence was not performed as increased refinement led to prohibitive computational runtimes on the systems available for this work. It is noted that the cell size utilized in these computations are consistent with the level of refinement commonly used to solve practical problems of interest.

The anvil in the classic configuration was modeled as a rigid structure, where the impact interface was modeled as frictionless since the local tangential velocity component at these material interfaces are unimpeded. In CTH it is not straightforward to model such a condition for the symmetric configuration, which is a particular difficulty common among Eulerian codes. The local velocity components along deformable material interfaces are equated, thus an infinite friction interface condition was modeled in the symmetric configurations. For both classic and symmetric configurations, the soda-lime glass rods were modeled using the SWRIG model with constants given in Table 2.

The following procedure was used in order to compare the experimental and computational images. The high speed photographs from the experiments show an envelope of the failed glass through the entire cross-section of the rod due to the transparency of glass. The images from the simulations correspond to isosurfaces of failure. These isosurfaces similarly show both internal and surface failure. It is noted that the possibility of shadows artificially appearing as damage in the experimental images is not possible in the computational results.

\section{Classic Configuration}

Sequential images during impact of specimen SL5 at a velocity of $370 \mathrm{~m} / \mathrm{s}$ in the classic configuration for both the experiment and simulation are shown in Fig. 11. Computational results are directly compared to high speed imaging using contours that indicate failed material as black,

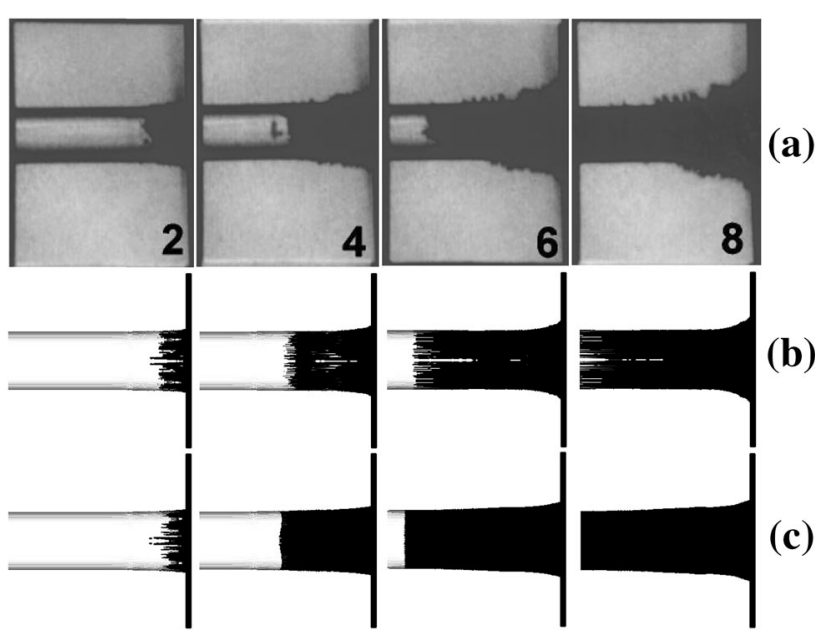

Fig. 11 Comparison of SL5 failure sequence: a high-speed photographs [14] and computational results for $\mathbf{b}$ baseline and $\mathbf{c}$ reduced interior strength simulations. Labels indicate time $(\mu \mathrm{s})$ after impact 
while intact glass is shown in white. In these images, the glass rod is initially traveling from left-to-right at the specified impact velocity and the anvil is seen as the thin black vertical strip on the right. Figure 11a are high speed photographs taken during the test by Willmott and Radford, while Fig. $11 \mathrm{~b}$ are corresponding CTH simulation results using the soda-lime glass model and parameters described previously. To study the effect that over-estimating the glass strength would have on the predictions, an additional simulation was performed with a reduced strength of interior material. Specifically, the interior strength was set equal to that of surface material $\left(D_{\text {int }}=D_{\text {surf }}\right)$ to bound the interior strength. The originally estimated interior strength represents an upper bound based on the available experimental data, while this assumed reduced strength likely represents a lower bound. The results from this reduced interior strength computation are shown in Fig. 11c. The number labels in the photographs indicate the time $(\mu \mathrm{s})$ after impact for each set (experimental and computational) of images. The time after impact, orientation, and scale of the numerical and experimental images are approximately identical.

The failure front is seen to have traveled a short distance from the impact plane in both the photograph (Fig. 11a) and the baseline computation (Fig. 11b) at $2 \mu$ s. The onset of very slight flaring of the specimen at the impact plane is also evident in both results at this time. Propagation of the failure front down the length of the rod, from right to left, is seen in the photographs at 4,6 , and $8 \mu \mathrm{s}$. The corresponding sequence from the baseline simulation shows the predicted position, and hence velocity, of the failure wave matches the experiment well. Finally, it is noted that the failure front has traveled beyond the field of view in the photographs and baseline simulation at $8 \mu \mathrm{s}$.

The radial expansion and flaring seen at the impact plane for these experiments is due to comminuted material being forced radially outwards rather than plastic deformation of intact material as is typically observed with metals under these conditions. This radial flaring is seen to increase during the progression of impact in both the experimental and baseline computation results. However, the extent of expansion seen in the experiment is significantly larger than was predicted. Additionally, the formation of discrete jets of comminuted material are seen in the photographs but not in the baseline simulation. These differences may indicate a limitation of the deterministic continuum model in capturing such a discrete and stochastic process that has been observed for glass [30, 31]. Mesh dependency is another possible contributing factor. Experimentally observed asymmetric failure front and expansion characteristics in addition to the small failure site seen ahead of the coherent front at $4 \mu$ s (and partially visible at $6 \mu \mathrm{s})$ are additional examples of the stochastic nature of the observed failure process that are not captured by the current glass model.

Willmott and Radford observed this localized failure in only a few frames during a limited number of their experiments. They postulated that this recurrence suggests a relatively high stress concentration due to coalescence of lateral release waves along the rod centerline or a high concentration of large flaws. The current glass model is parametrized assuming the rod initially contains some flaws concentrated on its surface (hence the reduced strength) and effectively zero interior flaws. The presence of significant internal flaws could be simulated by introducing localized regions of void or material having a reduced strength (such as that of reference or surface material). This would require the size, shape, and distribution of such flaws be known or assumed along with the assignment of an appropriate strength. Experimental data similar to that of Wereszczak et al. [31] might be used to estimate some of this type of initial flaw information.

The localized region of unfailed glass predicted along the centerline of the rod in the baseline computation (Fig. 11b) is likely a numerical artifact of the symmetry planes utilized in the computation. The texture of the predicted failure front that is evident in this baseline result and to a much lesser extent in the reduced strength computation (Fig. 11c) is presumably due the combination of numerical errors associated with the geometrical resolution of the deforming rod within the rectangular computational grid, advection artifacts, and wave interactions within the quarter-symmetric cylindrical geometry.

The reduced flaring and expansion in the baseline simulation results potentially indicates excessive strength for the soda-lime glass model. The excessive strength may be in-part due to the averaging procedure used in the estimation of the failed strength $\left(\sigma_{\max }^{f}\right)$ described previously (see Fig. 4). Uncertainties in the estimated surface $\left(D_{\text {surf }}\right)$ and interior $\left(D_{\text {int }}\right)$ strengths could result in excessive strength as well. The reduced interior strength computation in Fig. 11c displays increased radial expansion that reflects the experimentally observed phenomena somewhat better than the baseline result. Prediction of the failure front position in the reduced strength computation appears slightly low in the first frame and slightly high in later frames, but is otherwise similar to the original simulation and experimental results. This apparent delay in initial failure propagation might indicate a more appropriate strength somewhere between the two limiting cases assumed, but could also suggest the failure time $\left(t_{\text {fail }}\right)$ should be shorter than the estimated value of $0.6 \mu$ s. This latter possibility could also improve upon the prediction of the final transition to peak velocity in the plate impact 
wave profiles shown in Figs. 9 and 10. The observed flaw sensitivity discussed above could also play a role here, where the presence of more significant flaws would be expected to increase incremental rates of failure propagation. Although a more coherent failure front without symmetry plane artifacts is also seen in the reduced strength computation, the experimentally observed formation of discrete radial jets is still not predicted.

Figure 12 shows the observed and predicted state of the glass rod at the final frame of the image sequence at $11 \mu \mathrm{s}$ after impact for two nearly identical experimental cases. An image of specimen SL4 (Fig. 12a) is shown in addition to specimen SL5 (Fig. 12b) to illustrate some of the variability observed among experiments (impact velocities of 379 and $370 \mathrm{~m} / \mathrm{s}$, respectively) due in part to the aforementioned stochastic characteristics. The photographs of these two specimens are generally similar except for some differences in jets and radial expansion. Specifically, larger radial expansion is seen in SL5 than SL4 in terms of radial extent at the impact plane as well as along the rod length. Also, specimen SL4 displays more extensive discrete jets.

The baseline simulation result for SL5 at $11 \mu$ s is shown in Fig. 12c, where an under-prediction of radial expansion is seen without evidence of the jets observed in the photographs. Progressively increasing flaring at the impact plane is evident when one compares the baseline prediction at $8 \mu$ s (Fig. 11b) and $11 \mu$ s (Fig. 12c), which is roughly consistent with the progression observed in the photographs.

The result from the reduced interior strength simulation at $11 \mu \mathrm{s}$ is shown Fig. 12d. The primary difference between the reduced strength and the baseline simulation is the amount of radial expansion. Slightly more flaring localized at the impact plane is seen in the baseline result while the reduced strength result shows more expansion along the length of the rod, with some slight bulging a small distance from the impact plane that resembles the general shape of SL5 in the photograph. The interior strength reduction demonstrates the change in predicted response that may be achieved with a reasonable modification to a relatively uncertain SWRIG parameter estimated for soda-lime.

\section{Symmetric Configuration}

Sequential images during symmetric impact of specimen SL8 at a velocity of $391 \mathrm{~m} / \mathrm{s}$ are shown in Fig. 13. In this figure, the stationary rod is on the right and the impacting rod is traveling left-to-right. As with the classic configuration presented previously, Fig. 13a shows high speed photographs taken during the test by Willmott and Radford, while Fig. 13b shows the corresponding baseline CTH simulations results. At $3 \mu \mathrm{s}$, failure fronts originating from the plane of impact (the contact interface between the two rods) are seen to have traveled a short distance along each of the glass rods in both the photograph (Fig. 13a) and the baseline computational result (Fig. 13b). Significant propagation of the failure front down the length of the rods has occurred at $6 \mu \mathrm{s}$. At this time, the failure front has reached or exceeded the field of view for the impacting (left) rod while the failure front in the stationary (right) rod has roughly doubled in axial extent as compared to the image at $3 \mu \mathrm{s}$. The baseline predicted failure front shows some increased coherence and propagation later in time, but much less than that of the experiment. The same discrepancy is evident when the baseline computational and experimental results are compared at $9 \mu \mathrm{s}$. A possible explanation for this discrepancy is excessive glass strength (intact and failed), as was also indicated in the simulation results for the classic configuration.

The validity of this explanation is again examined through the impact sequence predicted from the reduced interior strength computation shown (Fig. 13c). A highly coherent failure front is seen in the reduced strength sequence, which is a significant improvement over the baseline result. Prediction of the failure front position in the reduced strength computation appears slightly overpredicted throughout the entire event. Thus, the reduced

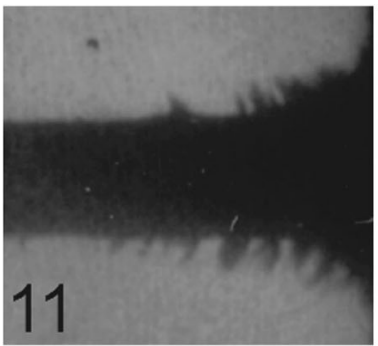

(a)

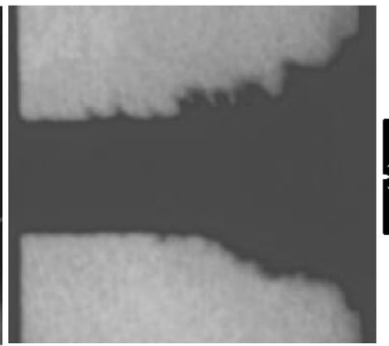

(b)

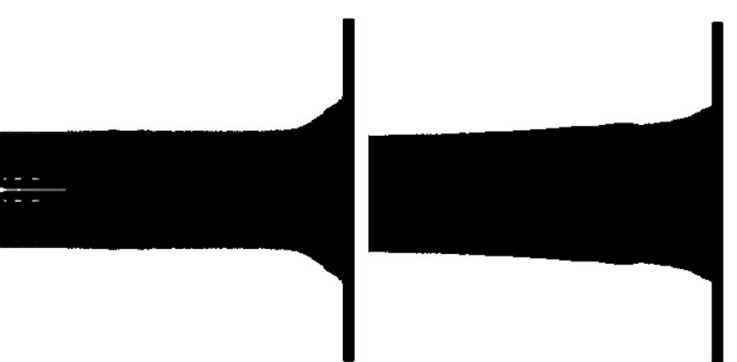

(c) (d)

Fig. 12 Deformation and failure characteristics $11 \mu$ s after impact: photographs [14] of a SL4, b SL5, computational results for $\mathbf{c}$ baseline and d reduced interior strength simulations 

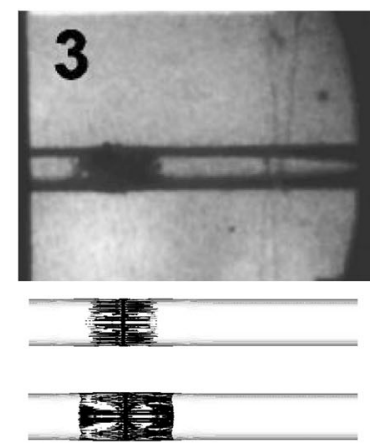
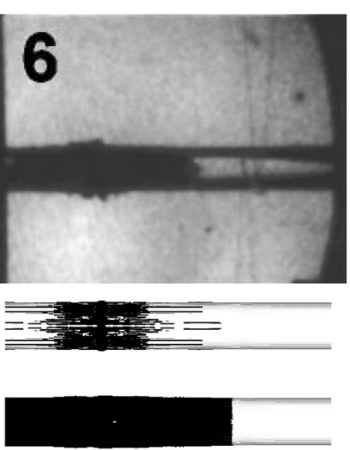
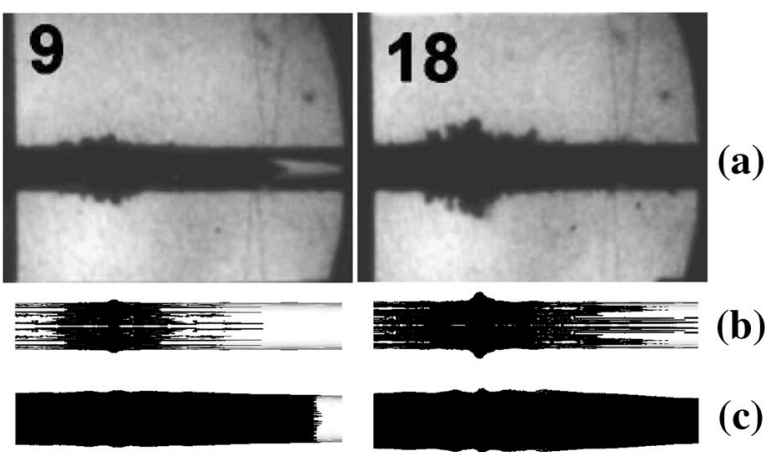

Fig. 13 Comparison of SL8 failure sequence: a high-speed photographs [14] and computational results for $\mathbf{b}$ baseline and $\mathbf{c}$ reduced interior strength simulations. Labels indicate time $(\mu \mathrm{s})$ after impact

strength result is seen to provide an upper bound to the experimental results while the baseline results provide more of a lower bound.

Significant radial expansion of comminuted glass initiates from the impact plane and is observed to develop during the course of the impact event in the experiments. The observed expansion is highly irregular and appears to display a less extensive and more discrete form in contrast to observations from the classic configuration. Reduced radial expansion in this configuration versus the classic configuration is seen in the baseline simulation, but the observed extent is under-predicted here as with the classic simulations. The axial distribution of radial expansion seen in the reduced strength result appears to yield only minor improvement, but the more discrete expansion at the impact plane in the experiment is not captured here. This suggests that the assumed strength of failed material may also be too high, as was noted above relative to the classic configuration. Both the baseline and reduced strength computations produced axially symmetric fracture that differs from the asymmetries observed experimentally. While the propagation of failure equally in all directions is promoted by the softening of neighboring material strength from surface or interior to reference (adjacent to failed), further propagation must be driven by continued inelastic loading. The load conditions (impact shock and wave interactions) and CTH model are axisymmetric for all cases, and lack the necessary asymmetries to produce the irregular structure seen in the experimental failure patterns. In the absence of continued yielding no additional plastic strain accumulates and thus failure stagnates. It may also be possible that too much energy is going into void insertion for tensile fracture instead of driving momentum transfer. Finally, the infinite friction rod interface condition modeled would be expected to impede radial expansion and flaring along the plane of impact.

Figure 14 shows the observed and predicted state of the glass rods (SL8) at the final frame of the image sequence for the symmetric configuration, $27 \mu$ s after impact. Comparison of the photographs at $18 \mu$ s (Fig. 13a) and $27 \mu$ s (Fig. 14a) reveals a progressive increase in the radial extent of expansion/jets. The baseline computation at $27 \mu \mathrm{s}$ is shown in Fig. 14b, where failure is seen to have exceeded the field of view. Similar to the comparison at $18 \mu \mathrm{s}$, significant flaring of the rods at the impact plane is predicted well, but extensive discrete radial jets are not captured in the baseline simulation (Fig. 14b).

The results of the reduced interior strength SL8 simulation $27 \mu$ s after impact are shown in Fig. 14c. The general observations of the reduced strength computation for SL5 also apply here for SL8; reduced interior strength yields increased radial expansion, however correlation to

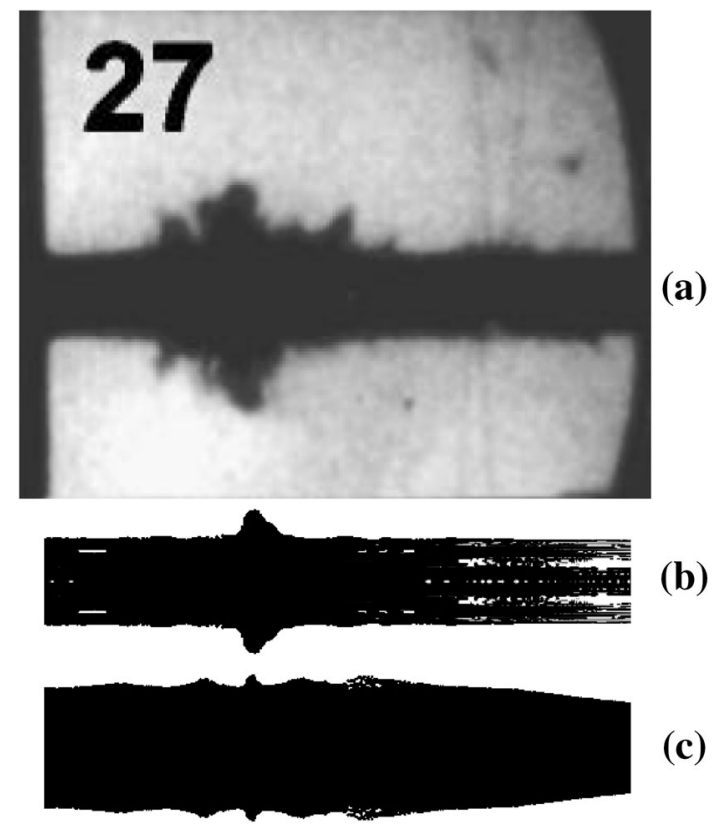

Fig. 14 Deformation and failure characteristics $27 \mu$ s after impact: photograph [14] of a SL8 and computational results for $\mathbf{b}$ baseline and c reduced interior strength simulations 
the experimental results are not as improved here as was observed for SL5. The extent of (maximum) radial expansion at the impact plane is about the same between the baseline (Fig. 14b) and reduced strength (Fig. 14c) computations. But, the reduced strength result shows much more expansion along the length of the rod, along with some more discrete features roughly comparable to those seen in the photograph. The maximum extent of expansion at the impact plane appears slightly under-predicted in the reduced strength computation, while expansion farther along the rod length appears over-predicted. While the interior strength reduction assumed here does not appear to resolve all of the observed discrepancies in this symmetric configuration, the potential for improvement with the SWRIG is indicated by the range in behavior possible with a reasonable modification to an estimated strength parameter for soda-lime.

\section{Failure Front Velocities}

Willmott and Radford calculated average failure front velocities from the front positions measured between sequential high-speed photographs during the first $7 \mu \mathrm{s}$ after impact; over distances up to $40 \mathrm{~mm}$ (4 rod diameters) from the impact plane. The error estimated in the calculated failure velocities from the experiments is a consequence of uncertainties in the measurements from the photographs and the stochastic nature of fracture.

This same method was also used to determine the predicted failure front velocities from the computations, which are shown in Table 5 along with the experimental results. Tolerances on the predicted failure velocities are due to sequential variations during the course of the impact event as well as uncertainties in the estimated front position. Predicted failure front velocities were calculated from each of the simulations presented (SL5 and SL8) in addition to simulations of the high velocity experiments (SL6 and SL9). Failure front velocities derived from the computations of SL5 and SL8 using reduced interior strength are shown in Table 5 in addition to those from the baseline computational results.
Predicted failure front velocities in the classic configuration are consistent with the experimentally determined values. The average velocities from the baseline computations are within approximately $10 \%$ of the experimentally determined values, with a consistent over-prediction indicated.

The average predicted velocities for the symmetric configuration are significantly lower than the experimental values. Large tolerances on these computational values are related to the prediction of a less coherent failure front and the associated difficulty in the determination of its position, as seen in Fig. 13. Including the leading edge of the incoherent failure streaks in the determination of failure velocity (the upper bounds of the indicated tolerances) yields predicted velocities in better agreement with the experiments.

The experimental data show higher failure front velocities in the symmetric configuration as compared to the classic, while the baseline computational results indicate the opposite trend. The variation in predicted failure front velocity during the course of the impact event is quite large for the symmetric computations (SL8 in particular). If the leading streaks of predicted failure are considered the actual failure front, the trend of higher velocities for the symmetric versus classic configurations is correctly predicted and this large variation is reduced to values consistent with the classic configuration computations.

It is expected that the infinite interface friction condition in the simulations of the symmetric configuration plays a role in the failure wave velocity predictions. This frictional condition contrasts the frictionless anvil interface in the classic simulations. This computational difference could in part explain the degraded symmetric configuration correlations and increased failure velocity uncertainty. The velocities derived from the reduced interior strength computations are an improvement, in that a higher failure velocity is predicted with the symmetric configuration. A lower strength could allow more shear deformation to occur at the material interface, thereby representing lower effective interface friction. Average velocities from the reduced strength computations are

Table 5 Measured [14] and predicted failure front velocities

\begin{tabular}{lllllll}
\hline Configurations & Specimens & Impact velocity $(\mathrm{m} / \mathrm{s})$ & Impact pressure $(\mathrm{GPa})$ & \multicolumn{2}{l}{ Failure front velocity $(\mathrm{km} / \mathrm{s})$} \\
\cline { 5 - 7 } & & & & Experiment & Baseline simulation & Reduced interior strength \\
\hline Classic & SL5 & $370 \pm 5$ & $3.8 \pm 0.1$ & $4.4 \pm 0.3$ & $4.9+0.6 /-0.9$ & $5.3+1.3 /-1.8$ \\
Classic & SL6 & $391 \pm 5$ & $4.1 \pm 0.1$ & $4.5 \pm 0.3$ & $4.9+0.6 /-1.4$ & - \\
Symmetric & SL8 & $391 \pm 5$ & $2.8 \pm 0.1$ & $5.6 \pm 0.6$ & $3.5 \pm 2.5$ & $5.7+1.8 /-2.2$ \\
Symmetric & SL9 & $485 \pm 1$ & $3.5 \pm 0.1$ & $5.3 \pm 0.5$ & $4.6+1.4 /-2.1$ & - \\
\hline
\end{tabular}




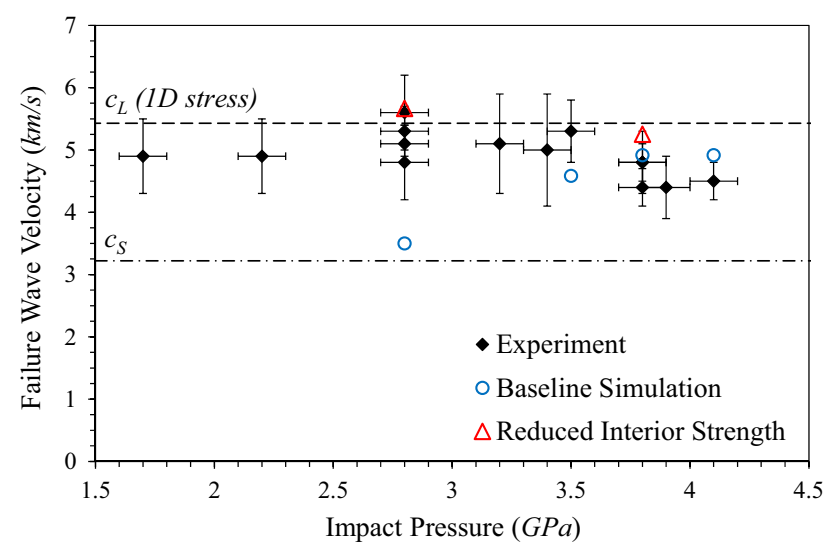

Fig. 15 Comparison of measured [14] and predicted pressuredependence of failure wave velocity

somewhat higher than in the experiments in general, although consideration of the tolerances shown implies the potential for closer agreement.

Predicted failure front velocities from Table 5 are shown graphically in Fig. 15 with all of the data from Willmott and Radford (Table 4). The longitudinal (1D stress) and shear wave velocities of soda-lime glass, $c_{L}$ and $c_{S}$, are also shown in Fig. 15 for reference. The average failure front velocities predicted in the baseline computations are in good overall agreement with the experimental data as a whole, with the exception of the under-prediction of the SL8 value. The computations assuming a reduced interior strength of soda-lime appear to significantly improve the consistency of the SL8 prediction with the test data, while having minimal adverse effect on the correlation for SL5. Failure front velocity appears to be approximately invariant with impact pressure and configuration (classic vs. symmetric) according to the experimental results. This invariance is roughly seen in the computational results as well. Overall, the computational results appear quite good when the correlation is viewed as a whole and considering the scatter seen in the experimental results.

\section{Conclusions}

A complete set of parameters for the glass model (SWRIG) by Holmquist and Johnson was developed characterizing commercially available soda-lime glass based primarily on experimental material characterization data available in open literature. The SWRIG model and developed parameters were used to model soda-lime glass under two different stress-deformation conditions where pressures and velocities span an order of magnitude (2.8-41 GPa,
$370-3220 \mathrm{~m} / \mathrm{s}$ ). These configurations specifically exercised key features of the glass model; the EOS, strength in both compression and tension, and damage/failure.

Computational results were overall found to be in good agreement throughout. Simulations of transmitted wave profile and reverberation experiments were found to capture much of the observed elastic and plastic shock and release response of soda-lime glass. Some of the more intricate aspects of the measured response, such as timedependent inelastic deformation, were not predicted particularly well. However, these unique characteristics of glass were not a primary focus in the development of the model parameters estimated for soda-lime glass. Simulation of failure during Taylor rod impact were also in generally good agreement with experimental results. The development of failure waves with reasonable propagation velocities were captured despite clear difficulties reproducing the observed radial expansion and discrete jet phenomena.

To the author's knowledge, this work represents the first application of this model to soda-lime glass. The highly complex behavior exhibited by glass and the uncertainties in many of the estimated model parameters pose significant challenges in realizing a true predictive capability for this material that is accurate under such a broad range of conditions. As such, improvements in computational results are expected to be possible with more work.

Experimental data characterizing the strength of glass from the uniaxial stress compression load path through the hydrostatic tensile regime is clearly lacking, hence the current model description of this behavior should be considered preliminary. The strong dependence of expansion characteristics of comminuted glass on the SWRIG model representation of soda-lime glass behavior was demonstrated in the Taylor impacts. Therefore, improvement upon the current results may be possible with some refinement of the soda-lime glass strength parameters better representing time-dependent loss of strength, modification to the assumed failed strength, and refinement of the failure strain in the damage model.

One aspect of glass response not directly addressed is the observed sensitivity to flaws and the statistical nature of fracture. Very high strengths have been observed in the absence of flaws while dramatic weakening has been seen in their presence. Specification of high internal tensile strength with lower strength of material on the surface and adjacent to failure is meant to loosely account for this flaw sensitivity. These provisions may be insufficient in the current description of soda-lime glass, however flaw-sensitivity is also a known difficulty with deterministic modeling of such stochastic processes. 
Acknowledgments This work was funded under Office of Naval Research (ONR) Contract N00014-14-C-0060. The authors gratefully acknowledge the support of Dr. RG Barsoum at ONR. Sandia National Laboratories is a multi-program laboratory managed and operated by Sandia Corporation, a wholly owned subsidiary of Lockheed Martin Corporation, for the US Department of Energy's National Nuclear Security Administration under Contract DE-AC0494AL85000

\section{References}

1. Holmquist TJ, Johnson GR (2011) A computational constitutive model for glass subjected to large strains, high strain rates and high pressures. J Appl Mech 78:051003

2. Anderson CE, Holmquist TJ (2013) Application of a computational glass model to compute propagation of failure from ballistic impact of borosilicate glass targets. Int J Impact Eng $56: 2-11$

3. Anderson CE (2009) Dwell and post-dwell penetration of long rods on borosilicate glass targets. In: Elert ML, Buttler WT, Furnish MD, Anderson WW, Proud WG (eds) Shock compression of condensed matter, vol 1195. AIP, New York, p 1447-1452. doi:10.1063/1.3295084

4. Anderson CE, Weiss CE, Chocron S (2009) Impact experiments into borosilicate glass at three scale sizes. Southwest Research Institute technical report 18.12544/018, San Antonio

5. Brar NS, Bless SJ, Rosenberg Z (1991) Impact-induced failure waves in glass bars and plates. Appl Phys Lett 59(26):3396-3398

6. Cagnoux J (1982) Shockwave compression of borosilicate glass up to 170 kbar. In: Shock compression of condensed matter, vol 78. AIP, New York, p 392-396. doi:10.1063/1.33346

7. Cagnoux J, Longy F (1988) Spallation and shock-wave behavior of some ceramics. J Phys Colloq 49(C3):C3-3-C3-10. doi:10. 1051/jphyscol:1988301

8. Chocron S, Anderson CE, Nicholls AE, Dannemann KA (2010) Characterization of confined intact and damaged borosilicate glass. J Am Ceram Soc 93(10):3390-3398

9. Forde LC, Proud WG, Walley SM, Church PD, Cullis IG (2010) Ballistic impact studies of a borosilicate glass. Int J Impact Eng 37:568-578

10. Nie X, Chen WW, Templeton DW (2010) Dynamic ring-on-ring equibiaxial flexural strength of borosilicate glass. Int J Appl Ceram Technol 7(5):616-624

11. Nie X, Chen W (2013) High-rate progressive failure of borosilicate glass under mechanical confinement at high temperatures. Exp Mech 53(1):67-75

12. Talladay TG, Templeton DW (2013) A computational comparison of high strain rate strength and failure models for glass. In: Proceedings of the 27th international symposium on ballistics, Freiburg, Germany, 22-26 April 2013, p 1630-1638

13. Bless SJ, Brar NS (2007) Failure waves and their effects on penetration mechanics in glass and ceramics. In: Horie Y (ed) Shockwave science and technology reference library, vol 2. Springer, Berlin, pp 105-141

14. Willmott GR, Radford DD (2005) Taylor impact of glass rods. J Appl Phys 97:093522

15. Alexander CS, Chhabildas LC, Reinhart WD, Templeton DW (2008) Changes to the shock response of fused quartz due to glass modification. Int J Impact Eng 35:1376-1385

16. McGlaun JM, Thompson SL (1990) CTH: a three-dimensional shock wave physics code. Int J Impact Eng 10:351-360

17. Johnson GR, Stryk RA, Holmquist TJ, Beissel RA (1997) Numerical algorithms in a Lagrangian hydrocode. Wright Laboratory technical report WL-TR-1997-7039. Wright Laboratory
18. Holmquist TJ, Johnson GR (2010) A computational constitutive model for glass subjected to large strains, high strain rates and high pressures. Southwest Research Institute technical report 18.12544/023, Minneapolis

19. Holmquist TJ, Johnson GR, Grady DE, Lopatin CM, Hertel Jr ES (1995) High strain rate properties and constitutive modeling of glass. In: 15th International symposium on ballistics, Jerusalem, Israel, 21-24 May 1995

20. Holmquist TJ, Rajendran AM, Templeton DW, Bishnoi KD (1999) A ceramic armor material database. TARDEC technical report 13754. US Army Tank Automotive Research, Development and Engineering Center, AMSTA-TR-R (MS 263), Warren

21. Alexander CS, Chhabildas LC, Templeton DW (2007) The Hugoniot elastic limit of soda-lime glass. In: Shock compression of condensed matter, vol 955. AIP, New York, p 733-738

22. Dannemann K, Anderson C, Chocron S, Spencer J (2011) Damage development in confined borosilicate and soda-lime glasses. TARDEC technical report 21945. US Army Tank Automotive Research, Development and Engineering Center, Warren

23. Sundaram S, Clifton RJ (1998) Flow behavior of soda-lime glass at high pressures and high shear rates. In: Shock compression of condensed matter, vol 429. AIP, New York, p 517-520

24. Clifton RJ, Mello M, Brar NS (1998) Effect of shear on failure waves in soda lime glass. In: Shock compression of condensed matter, vol 429. AIP, New York, p 521-524

25. Espinosa HD, Xu Y, Brar NS (1997) Micromechanics of failure waves in glass: I, experiments. J Am Ceram Soc 80(8):2061-2073

26. Simha CHM, Gupta YM (2004) Time-dependent inelastic deformation of shocked soda-lime glass. J Appl Phys 96(4):1880-1890

27. Bourne N, Millett J, Rosenberg Z, Murray N (1998) On the shock induced failure of brittle solids. J Mech Phys Solids 46(10): 1887-1908

28. Bless SJ, Brar NS, Rosenberg Z (1988) Strength of soda lime glass under shock compression. In: Schmidt SC, Holmes NC (eds) Shock waves in condensed matter, 1987. Elsevier Science, Amsterdam, pp 309-312

29. Rosenberg Z, Yaziv D, Bless S (1985) Spall strength of shockloaded glass. J Appl Phys 58(8):3249-3251

30. Wereszczak AA, Johanns KE, Kirkland TP, Anderson Jr CE, Behner T, Patel P, Templeton DW (2006) Strength and contact damage responses in a soda-lime-silicate and a borosilicate glass. In: 25th Army science conference, Orlando, FL, 27-30 November

31. Wereszczak AA, Kirkland TP, Ragan ME, Strong KT Jr, Lin H-T (2010) Size scaling of tensile failure stress in a float soda-limesilicate glass. Int J Appl Glass Sci 1(2):143-150

32. Grady DE, Chhabildas LC (1996) Shock wave properties of soda lime glass. In: Iyer KR, Chou SC (eds) Proceedings of 14th US Army symposium on solid mechanics, Myrtle Beach, SC. Battelle Press, Columbus

33. Dandekar DP (1998) Index of refraction and mechanical behavior of soda lime glass under shock and release wave propagations. J Appl Phys 84(12):6614

34. Dandekar DP, Beaulieu PA (1995) Failure wave under shock wave compression in soda lime glass. In: Murr LE, Staudhammer KP, Myers MA (eds), Proceedings of 1995 international conference on metallurgical and materials applications of shock-wave and high-strain-rate phenomena, El Paso, TX. Elsevier Science, Amsterdam, Netherlands, p 211

35. Ji H, Keryvin V, Rouxel T, Hammouda T (2006) Densification of window glass under very high pressure and its relevance to Vickers indentation. Scr Mater 55:1159-1162

36. Pilkington North America, Inc. (2012) Properties of soda-limesilica float glass. Technical information ATS-129, 26 April 
37. Barker LM (1972) Laser interferometry in shock-wave research. Exp Mech 12(5):209-215

38. Hertel Jr ES, Kerley GI (1998) CTH reference manual: the equation of state package. Sandia National Laboratories report SAND98-0947, Albuquerque
39. Group GMX-6 (1969) Selected Hugoniots. Los Alamos Scientific Laboratory report LA-4167-MS, Los Alamos

40. Steinberg DJ (1996) Equation of state and strength properties of selected materials. Lawrence Livermore National Laboratory report UCRL-MA-106439 Change 1, Livermore 